\title{
A sensitive and smartphone colorimetric assay for the detection of hydrogen peroxide based on antibacterial and antifungal matcha extract silver nanoparticles enriched with polyphenol
}

\author{
Ezgi Tan ${ }^{1} \cdot$ İbrahim Mizan Kahyaoğlư ${ }^{2}$ Selcan Karakuş ${ }^{1}$
}

Received: 7 July 2021 / Revised: 2 August 2021 / Accepted: 9 August 2021 /

Published online: 14 August 2021

(C) The Author(s), under exclusive licence to Springer-Verlag GmbH Germany, part of Springer Nature 2021

\begin{abstract}
Current trends in scientific studies focus on the development of smartphone-based biosensors via green nanoparticle for clinical diagnosis, food, and environmental monitoring. In this study, we developed a novel portable smartphone-based biosensor via green dendrimer-coated matcha extract/silver nanoparticles (ME-Ag NPs) enriched with polyphenol for detecting hydrogen peroxide $\left(\mathrm{H}_{2} \mathrm{O}_{2}\right)$. Also, we investigated the biological evaluation of the nanostructure as a safe preservative for use in biomedical applications. Ag NPs were prepared using a green sonochemical method and were characterized to determine surface and chemical properties by different techniques such as scanning electron microscopy-energy-dispersive X-ray, transmission electron microscope, Fourier transform infrared spectroscopy, atomic force microscopy, X-ray diffraction, and Brunauer-Emmett-Teller. Furthermore, antimicrobial and antifungal properties of ME-Ag NPs were investigated against pathogenic microorganisms such as Staphylococcus aureus, Pseudomonas aureginosa, Escherichia coli, Candida albicans, and Aspergillus brasiliensis. The experimental sensor methodology was based on the detection of $\mathrm{H}_{2} \mathrm{O}_{2}$ by analysis of images of novel silver nanostructure-coated papers and processing of color histograms with a RGB (red-green-blue) analyzer software. Consequently, the smartphone-based biosensor exhibited high sensitivity with detection limits of $0.82 \mu \mathrm{M}$ response time of $5 \mathrm{~s}$. The smartphone-based biosensor via ME-Ag NPs provided a rapid and selective detection of $\mathrm{H}_{2} \mathrm{O}_{2}$.
\end{abstract}

Keywords Silver nanoparticle enriched with polyphenol - Surface characterization · Smartphone biosensor $\cdot$ Antimicrobial agent $\cdot$ Nanostructure

Ezgi Tan

ezgi.tan@iuc.edu.tr

Extended author information available on the last page of the article 


\section{Introduction}

The monitoring and detection of $\mathrm{H}_{2} \mathrm{O}_{2}$ have a significant role in biological organisms and a broad range of industrial applications [1]. For the determination of $\mathrm{H}_{2} \mathrm{O}_{2}$, different methods such as chromatography, electroanalysis, colorimetry, and fluorometry have been reported [2, 3]. Instead of bulky and expensive methods such as spectrophotometer, which are widely used to detection of target biochemical analytes, easy to use, portable, and inexpensive smartphone-based methods offer great advantages [4] In this case, it is significant to develop easyto-use and low-cost smartphone-based methods to detect $\mathrm{H}_{2} \mathrm{O}_{2}$ with a high sensitivity and rapid detection at low concentration. In the last two decades, scientific studies focus on smartphone-based sensor technologies, which have great potential in studies for different purposes such as high-quality medical services, food control, diagnostic technologies, and environmental monitoring [5-7]. In particular, the use of smartphones as detectors has increased, which makes the detection method considerably practical and economical and facilitates its commercialization and spread in modern sensor technology. The smartphone is used as a detector, reflecting the colorimetric analysis using an image software-based histogram algorithm. The methodology of smartphone-based sensor is based on a highresolution camera and image software using mobile sensing approaches under a smartphone's flashlight or an external light source [3]. The digital image colorimetry method on a smartphone is known a fast and low-cost analysis method using a RGB analyzer software. A target analyte is (metal, drug, microorganism, and biomarkers) measured by color analysis of the digital image obtained using the built-in camera by the digital image colorimetry method [8-10].

Recently, nanostructures have been widely used in the development of electrochemical biosensors to improve the biocompatibility, sensitivity, reproducibility, catalytic activity, and signal amplification [11-13]. With the remarkable advances and developments in nanotechnology, various nanomaterials such as nanolayers [14], quantum dots [15], NPs [16, 17], carbon nanotubes [18], nanofiber [19], and nanocomposite [20] have been reported to be used in the development of electrochemical biosensors. Our aim was to develop the disposable colorimetric nanobiosensor paper and evaluate the color change with a smartphone. In this context, we designed a new colorimetric biosensor by integrating the nanostructure with a smartphone platform to apply the promising material in $\mathrm{H}_{2} \mathrm{O}_{2}$ determination. In this study, we developed a novel smartphone-based biosensor via green dendrimer-coated matcha extract/silver nanoparticles (ME-Ag NPs) enriched with ployphenol for detecting $\mathrm{H}_{2} \mathrm{O}_{2}$ based on digital RGB spectral responses. The smartphone-assisted method was based on nanoprobe-coated filter paper using RGB colorimetric approach. Additionally, the strategy of using smartphone-based biosensor to detect $\mathrm{H}_{2} \mathrm{O}_{2}$ has not been reported and has unique advantages such as green, low-cost, portable, sensitivity, and easy to use.

In addition, biological evaluation of novel ME-Ag NPs was performed in this study. As known, current trends in scientific studies focus on the control the spreading and to prevent infections of microscopic organisms known as 
pathogens - such as coronavirus, cholera and plague or parasites [21-23]. One of the most promising developments of nanotechnolgy is the evaluation of preservative efficacy of nanoadditives, which have been gaining attention for preventing from pathogenic microbial contamination during the novel fabrication of cosmetics [24-27]. Recently, metal/metal oxide-based nanoformulations are promising nanosized protectants against several pathogens in food, cosmetic, agriculture and drug with their excellent antimicrobial and antifungal properties [28-32]. Among these materials, green synthesized silver-based nanostructures are widely used to control bacterial growth in biomedical applications [33]. Ag-based nanostructures have a significant performance to penetrate cell walls of bacterial, changing the chemical structure of cell membrane of bacteria and even resulting in bacterial death due to their small size and biocompatibility, and excellent physicochemical properties [34, 35]. According to technological development and previous studies, several novel silver-based nanoformulations have been fabricated using different stabilizers such as chitosan [36], alginate [37], poly (methyl methacrylate) (PMMA) [38], polyethylene glycol-polyvinyl alcohol (PEG-PVA) [39], PVA/gum acacia [40], polyvinylpyrrolidone (PVP) [41], polylactic acid (PLA) [42], clay [43], and plant extract [44]. In particular, several studies have been proposed to explain the physicochemical and biological properties of plant extractbased Ag NPs in the literature. However, some disadvantages have been studied to control the miscibility, dispersion stability, and agglomeration of Ag-based formulation in liquids [45, 46]. With these disadvantages, desired outcomes have not been completely achieved. To overcome these problems, Ag NPs have been developed due to their large surface area, excellent dispersion due to then narrow size distribution, stable dispersion in aqueous solutions, and highly branched shaped structures to improve antimicrobial and antifungal properties. Another prominent aspect of this study was the use of low-cost, biodegradable matcha, which is grown in many countries of the world, as a green stabilizer in the synthesis of silver nanostructures.

For this purpose, we synthesized the hyperbranched unimolecular ME-Ag NPs (generation 3, G3) by the green and one-step sonochemical via a self-assembly process.

The surface characterization and functional groups of ME-Ag NPs were determined by different techniques such as SEM-EDX, TEM, FTIR AFM, XRD, and BET. Furthermore, we focused on the development of the hyperbranched dendrimer-coated silver nanoparticles (ME-Ag NPs) to establish a novel preservative nanoformulation for antifungal agents against microorganisms and mold-yeast in biomedical and cosmetic applications. We determined the preservative efficacy of ME-Ag NPs against pathogenic microorganisms such as S. aureus, P. aeruginosa, E. coli, C. albicans, and A. brasilliensis with 1SO 11930:2012 guideline for the total aerobic mesophilic microorganism, and mold-yeast, respectively. Consequently, the biological results were examined comprehensively, the novel green nanostructure exhibited unique antifungal and antibacterial properties. Furthermore, the sensor results showed that the prepared smartphone-based biosensor is a promising system for development of the smartphone-based sensors for other contaminants. 


\section{Materials and methods}

\section{Materials}

Matcha powder (Camellia sinensis) purchased from Arifoğlu Company (Turkey). Sodium hydroxide, isopropyl alcohol, ethanol, ethyl acetate, and ammonia were purchased from Merck Company. Whatman ${ }^{\circledR}$ qualitative filter paper (diam. $25 \mathrm{~mm}$ ) and silver nitrate $(\geq 99.0 \%)$ were purchased from Sigma-Aldrich Company (Germany). Ascorbic acid (L-Ascorbic acid 99\%), dopamine hydrochloride (98\%), progesterone (4-Pregnene-3,20-dione, $\geq 99 \%$ ), and glucose (D-(+)-Glucose monohydrate, 99.0\%) were obtained from Sigma-Aldrich Company Ltd. (St Louis, USA). We used a sterile syringe filter with a 0.22 -micron retention and polyvinylidene difluoride (PVDF) membrane. Ultrapure water was obtained from Merck Millipore Milli-Q® Integral 5. All chemicals and reagents were used without further purification.

\section{Preparation of ME}

First, ME powder was cleaned with distilled water to remove all impurities and then dried to constant weight at $50{ }^{\circ} \mathrm{C}$ in a vacuum heater for $2 \mathrm{~h}$ to remove residual moisture. Second, $10 \mathrm{~g}$ of the matcha powder was added to $500 \mathrm{ml}$ of distilled water for 7 days at $25{ }^{\circ} \mathrm{C}$ in the dark medium at stable position. Finally, the solution was filtered using a sterile syringe filter to obtain ME. All samples were stored in sterile polypropylene containers until use at $25^{\circ} \mathrm{C}$.

\section{The self-assembly sonofabrication of the Ag NPs enriched with polyphenol}

The self-assembly sonochemical synthesis of ME-Ag NPs consisted of four steps. The first step, $0.42 \mathrm{~g}$ of $\mathrm{AgNO}_{3}$ was dissolved in $250 \mathrm{ml}$ of distilled water. Then, the solution was stirred for $10 \mathrm{~min}$ at $25^{\circ} \mathrm{C} .0 .2 \mathrm{~g}$ of $\mathrm{NaOH}$ was dissolved in $125 \mathrm{ml}$ of distilled water and stirred for $10 \mathrm{~min}$ at $25{ }^{\circ} \mathrm{C}$. The second step, $50 \mathrm{ml}$ of the $\mathrm{ME}$ and $100 \mathrm{ml}$ of $\mathrm{AgNO}_{3}$ solution, was sonicated for $5 \mathrm{~min}$ (amplitude frequency: $30 \%$ ). The third step, $1 \mathrm{ml}$ of $\mathrm{NaOH}$ solution was added slowly to the solution. We observed the color change of the sample. We assumed that ME had a role as a green reducing agent for reducing $\mathrm{Ag}^{+}$to $\mathrm{Ag}^{\mathrm{o}}$ by changing yellow to brown. Finally, ME-Ag NPs were filtered using a sterile syringe filter and stored in a sterile container until use at $25^{\circ} \mathrm{C}$ in vacuum desiccator.

\section{Surface characterization of the nanostructure}

We used different advanced techniques such as scanning electron microscopy (SEM) - energy-dispersive X-ray (EDX), transmission electron microscope (TEM), Fourier transform infrared (FTIR) spectroscopy, atomic force microscopy (AFM), X-ray diffraction (XRD), and Brunauer-Emmett-Teller (BET) to characterize the nanostructure. The SEM-EDX (FEI QUANTA 450) (TedPella, double-coated, $8 \mathrm{mmW} \times 20 \mathrm{~mL}$ ) was used to determine the surface morphology of the ME-Ag NPs 
with a 6-10 mm working distance, 0-130 Pa pressure, and voltage of 7-10 kV under low vacuum medium. Transmission electron microscope (TEM) (Hitachi HighTech HT7700) was used to visualize the ME-Ag NPs in a high vacuum mode at $100 \mathrm{kV}$. FTIR (Spectrum Two, Perkin Elmer,) was used to observe the functional groups of the samples ( $\mathrm{KBr}$ powder) in the $4000-400 \mathrm{~cm}^{-1}$ frequency range with a resolution of $4 \mathrm{~cm}^{-1}$ and eight scans. The crystal structure and the mean size of crystallites of the ME-Ag NPs were determined by the XRD technique with $\mathrm{Cu} \mathrm{K} \alpha$ radiation at $40 \mathrm{kV}$ and $15 \mathrm{~mA}$. The Brunauer-Emmett-Teller (BET) specific surface area of the sample was determined using a Micromeritics ASAP 2020. Absorbances of reference (NPs) and sample (NPs + analyte) were measured using a double-beam $\mathrm{UV}-\mathrm{V}$ is spectrophotometer (T+80, PG Instrument) with a UVWin 5 Software.

\section{Calculations part}

The crystalline size of ME-Ag NPs was calculated using different XRD mathematical models such as the Scherrer, Williamson-Hall, and modified Scherrer equations, respectively (Eqs. 2.1-2.3) [47, 48]. The formulas of all models are given in Table 1.

\section{Storage stability of the nanostructure}

In this study, the storage stability of the synthesized ME-Ag NPs was evaluated under ultraviolet light. Five milliliters of ME-Ag NPs solution was irradiated at $365 \mathrm{~nm}$ for $2 \mathrm{~h}$. We experimentally examined the effect of ultraviolet irradiation on the storage stability of the synthesized ME-Ag NPs by measuring the viscosity. Experimental rheological measurements were performed in triplicate.

\section{Antimicrobial and antifungal assays}

In this study, the synthesized hyperbranched ME-Ag NPs enriched with polyphenol (generation 3, G3) were performed for antimicrobial and antifungal effect against different pathogenic microorganisms such as staphylococcus aureus ( $S$. aureus, NCTC 10,788/Lot 0,350,520,029), Pseudomonas aureginosa (P. aeruginosa, ATCC 9027/Lot 3270513), Escherichia coli (E. coli, ATCC 8739/Lot 4835151), Candida albicans (C. albicans, NCPF 3179/Lot040920020), and Aspergillus brasilliensis (A. brasilliensis, NCPF 2275/Lot 020620065).

Table 1 The formulas of Scherrer, Williamson-Hall, and modified Scherrer models

\begin{tabular}{llc}
\hline Formula & Model & Eq. \\
\hline$D_{p}=\frac{0.94 \cdot \lambda}{\beta \cdot \cos \theta}$ & Scherrer equation & (2.1) \\
$\beta \cos \theta=\frac{0.94 \cdot \lambda}{D_{p}}+4 \varepsilon \sin \theta$ & Williamson-Hall equation & (2.2) \\
In $(1 / \cos \theta)=\operatorname{In} \frac{0.94 \cdot \lambda}{D_{p}}+\operatorname{In} \beta$ & Modified Scherrer equation & (2.3) \\
\hline
\end{tabular}

Dp average crystallite size, $\beta$ line broadening in radians, $\theta$ Bragg angle, $\lambda \mathrm{X}$-ray wavelength 


\section{Challenge test}

The challenge test was performed using the standard NF EN 1SO 11930:2012 guideline to evaluate the microbiological safety and level of biological activity of the preservative ME-Ag NPs (Table 2). We examined by inoculation of the synthesized ME-Ag NPs with pathogenic microorganisms at a concentration range from $10^{5}$ and $10^{6} \mathrm{CFU} \mathrm{g}^{-1}$ corresponded to the colony-forming units per $\mathrm{g}$ of the synthesized ME-Ag NPs.

Different pathogenic microorganisms were used such as Staphylococcus aureus (S. aureus, NCTC 10788/Lot 0350520029), Pseudomonas aureginosa (P. aeruginosa, ATCC 9027/Lot 3270513), Escherichia coli (E. coli, ATCC 8739/Lot 4835151), Candida albicans (C. albicans, NCPF 3179/Lot040920020), and Aspergillus brasilliensis (A. brasilliensis, NCPF 2275/Lot 020620065) and analyzed for challenging test of the synthesized ME-Ag NPs. All pathogenic microorganisms were issued from official collection of microbial strains of The European Union (EU) that ensures the reproducibility of the challenging tests. The inoculated ME- Ag NPs was held at temperature ranges from 20 to $25{ }^{\circ} \mathrm{C}$ in the dark medium for 28 days. The viability assessment was performed at suitable time intervals ( $\mathrm{T} 0=0$ days (immediately after collection); T7 $=7$ days; $\mathrm{T} 14=14$ days; and T28 $=28$ storage days) with to the number of viable microorganisms. The preservative effectiveness test results were expressed in terms of the criteria A and B described in Table 3.

In particular, the microbiological criteria were defined from decrease in logarithmic reduction scale 10 (log scale 10) of microorganism concentration from the measured inoculum using challenge test (according to the standard NF EN ISO 11930) for the evaluation of the antimicrobial protection of a cosmetic product. The result of challenge test gives two types of criteria such as a target (the "A" criterion) and an acceptable level (the "B" criterion). Finally, "no increase" was defined as not much more than $0.5 \log 10$ units higher than the previous value measured.

\section{Preparation of smartphone-based hydrogen peroxide biosensor}

The preparation steps of the biosensor arrays were as follows.

Table 2 Experimental challenge test conditions

\begin{tabular}{lll}
\hline Microorganisms & Culture medium & $\begin{array}{l}\text { Experimental incu- } \\
\text { bation temperature } \\
\left({ }^{\circ} \mathrm{C}\right)\end{array}$ \\
\hline S. aureus, NCTC 10788/Lot 0350520029 & Tryptic soy agar & $30-35$ \\
P. aeruginosa, ATCC 9027/Lot 3270513 & Tryptic soy agar & $30-35$ \\
E. coli, ATCC 8739/Lot 4835151 & Tryptic soy agar & $30-35$ \\
C. albicans, NCPF 3179/Lot040920020 & Saboraud 4\% dextrose agar & $20-25$ \\
A. brasilliensis, NCPF 2275/Lot 020620065 & Saboraud 4\% dextrose agar & $20-25$ \\
\hline
\end{tabular}




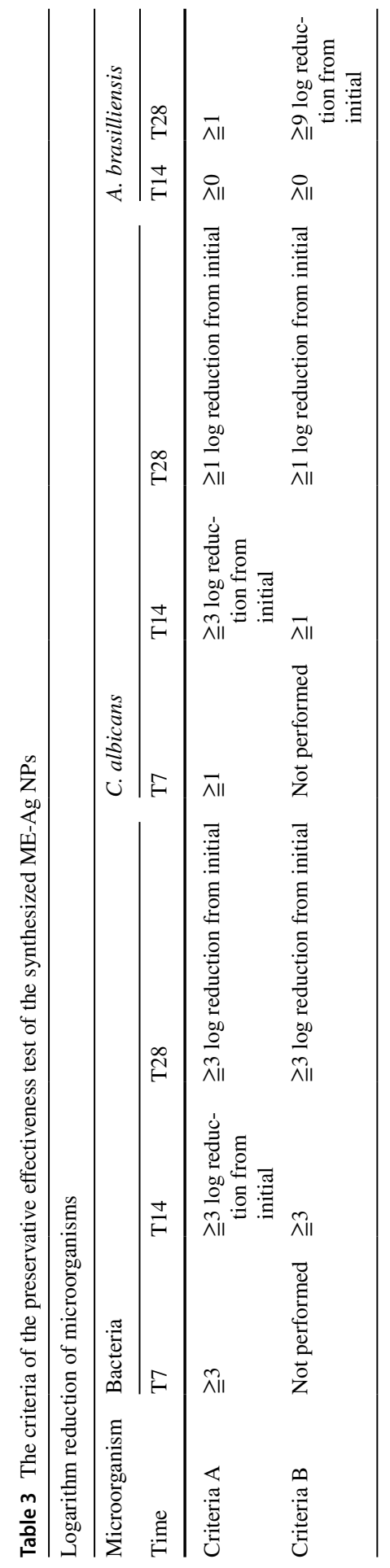


(1) colorimetric solutions of ME-Ag NPs were dropped in different concentrations on a Whatman ${ }^{\circledR}$ qualitative filter paper using an Eppendorf® Research $®$ plus pipette $(0.1-2.5 \mu \mathrm{L})$, constructing a $2 \mathrm{~cm}$ diameter circular area of color-sensitive sensor array and (2) the sensor arrays were dried for $10 \mathrm{~min}$ and deposited in a sterile polyethylene bag before use. (3) $\mathrm{H}_{2} \mathrm{O}_{2}$ solutions in the range from 0.05 to $20 \mu \mathrm{M}$ were dropped on a ME-Ag NP-coated filter paper. (4) The images all samples were taken at room temperature with a smartphone (Casper F20) with a resolution of $1600 \times 1200$ pixels. Histograms of images of samples were obtained using a software (ImageJ 1.51q software) and analyzed the distribution of RGB values of samples. All experiments were performed in triplicate $(n=3)$.

\section{Selectivity analysis}

The selectivity of the process was performed by detecting the effects of different analytes such as $\mathrm{H}_{2} \mathrm{O}_{2}$, isopropyl alcohol, ethanol, ethyl acetate, ammonia, ascorbic acid, dopamine hydrochloride, progesterone, and glucose in the response of the nanoprobe as in "Preparation of smartphone-based hydrogen peroxide biosensor" section.

\section{Quantitative recognition assisted using a smartphone via the RGB analysis}

In this study, we developed the novel portable smartphone-based biosensor via green ME-Ag NPs for detecting of $\mathrm{H}_{2} \mathrm{O}_{2}$. Furthermore, the detection mechanism of NPs biosensor was based on the characteristic color change of the nanocoated paper sensor, which was responded selective and rapid to target analyte using smartphone images of sample. Also, we detected the color change of samples and determined the $\mathrm{H}_{2} \mathrm{O}_{2}$ concentration quantitatively using a spectroscopic measurement technique due to reaction with the target analyte.

All samples were imaged using a smartphone under sun light. We put the smartphone in a plexiglass glass-based box $(8 \mathrm{~cm} \times 8 \mathrm{~cm}$ and a 3 -mm-thick clear acrylic sheets with a protective film) to keep it in a stable position. The colorimetric histograms of samples were determined to understand color differences in images using the RGB analysis with the software ImageJ. Euclidean distance $(\Delta E)$ values were calculated to observe the $\mathrm{H}_{2} \mathrm{O}_{2}$ quantitative detection in the range from $[0,0,0]$ and [255, 255, 255] RGB values (Eq. 2.4) [49]. According to RGB analysis, values of color were assigned in the range from 0 to 255 for absolute black and absolute white, respectively.

$$
\Delta E=\sqrt{\left(R_{i}-R_{0}\right)^{2}+\left(G_{i}-G_{0}\right)^{2}+\left(B_{i}-B_{0}\right)^{2}}
$$

where the subscripts $i$ and 0 denote the RGB value ( $R$ : red, $G$ : green, and $B$ : blue) of the ME-Ag NPs with and without the analyte, respectively. The correlation between $\Delta \mathrm{E}$ values and $\log \mathrm{H}_{2} \mathrm{O}_{2}$ concentration was obtained to determine the equilibrium time and colorimetric quantitative recognition. Furthermore, we calculated sensor 
response data for ME-Ag NPs sensor papers to investigate their performance for sensor applications (Eq. 2.5) [50].

$$
S=100 \frac{x_{c}-x_{0}}{x_{0}-x_{r e f}}[\%]
$$

where $\mathrm{S}$ : response in $\%, c$ : concentration, $x_{c}$ : sensor signal (RGB channels), $x_{0}$ : the value of $x(c=0)$ signal, and $x_{\text {ref }}:$ reference value. According to Eq. 2.6, we investigated the response of NPs paper sensor with the logarithm of the $\mathrm{H}_{2} \mathrm{O}_{2}$ concentration [50].

$$
S=m \log (c)+n[\%]
$$

where $m$ : constant and $n$ : response at low concentration.

Limit of detection (LOD) was known concentration of a component and calculated from calibration curve of the sample.

$$
L O D=3,3 \times \sigma / S
$$

where $S$ is the slope of the calibration curve and $\sigma$ is the standard deviation of the response.

\section{Statistical analysis}

All experimental results were obtained in triplicate and are given as the mean for each experiment. The analysis of variance (ANOVA) procedure was conducted in SPSS (version 16, Chicago) with a significance level of $P<0.05$.

\section{Results and discussion}

\section{Surface characterization of the nanostructure}

In this study, ME was used as a stabilizing agent for hyperbranched unimolecular ME-Ag NPs (generation 3, G3). Surface techniques were used to control the size, dispersion, and stability of Ag NPs. Surface morphology and particle size of ME and ME-Ag NPs (G3-Ag) were investigated by SEM technique. SEM images of (a) pure matcha powder, (b) ME-Ag NPs with 40.000 $\times$ magnification, (c) ME-Ag NPs with $160.000 \times$ magnification, (d) EDX spectra of ME-Ag NPs are given in Fig. 1. The SEM result showed that pure matcha powder had an agglomerated and irregular shaped (Fig. 1a) [51, 52]. Figure 1b shows the SEM images of third generation ME-Ag NPs encapsulated with Ag nanoparticles which were uniformly anchored spheroidal dispersed within the polyphenolic dendrimer branching network. The size of Ag nanoparticles was found the ranges from 10 to $30 \mathrm{~nm}$. According to SEM results, we showed the successful encapsulation of Ag nanoparticles within hyperbranched unimolecular polyphenolic dendrimer. The EDX spectra of ME-Ag NPs is shown in Fig. 1c. The characteristic absorption peaks of $\mathrm{Ag}^{\mathrm{o}}$ in the range of 1-2 

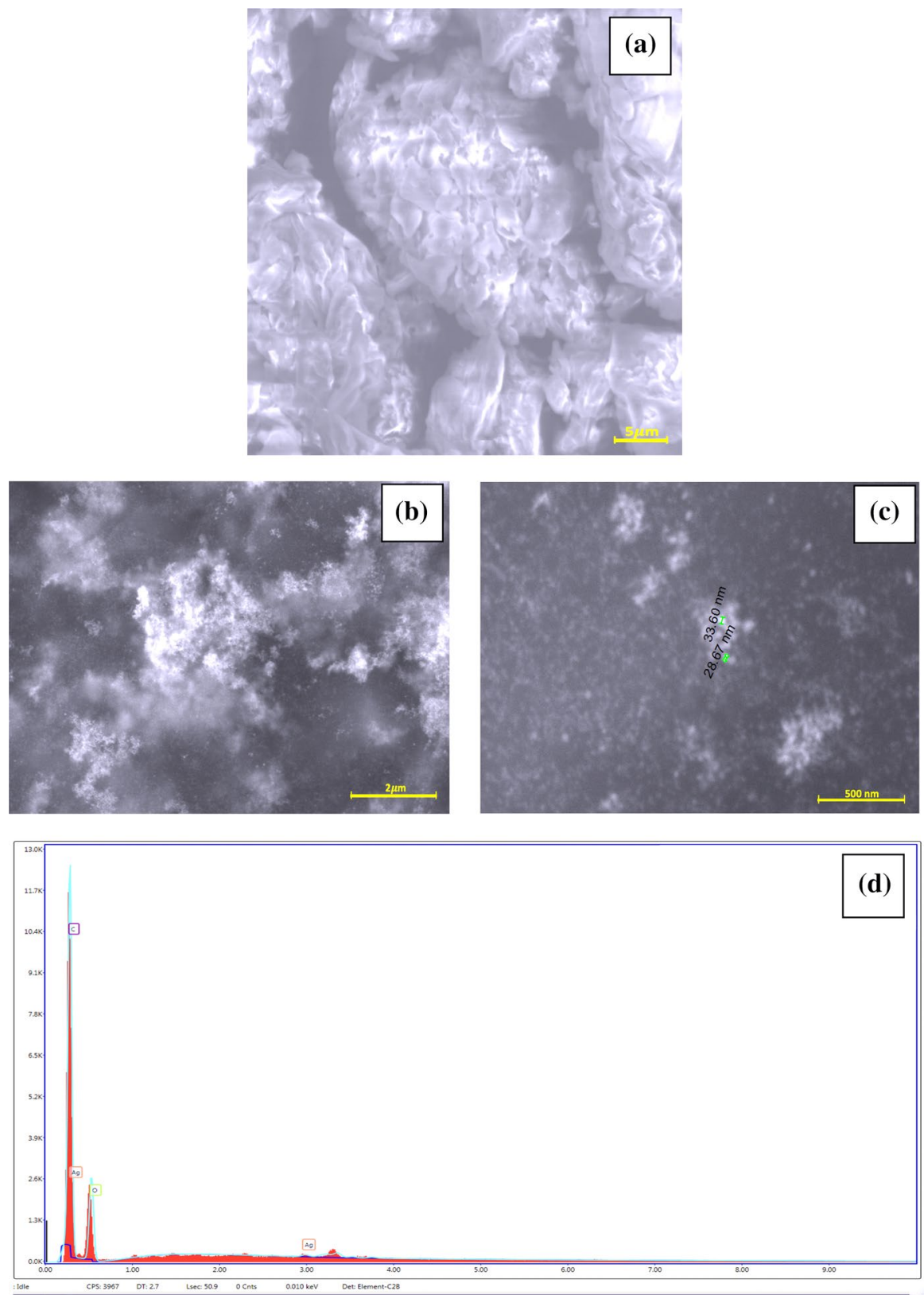

Fig. 1 SEM images of a pure matcha powder, b ME-Ag NPs with $40.000 \times$ magnification, $\mathbf{c}$ ME-Ag NPs with $160.000 \times$ magnification, $\mathbf{d}$ EDX spectra of ME-Ag NPs 
and $3 \mathrm{keV}$ were observed and the elemental EDX analysis also revealed $\mathrm{C}, \mathrm{O}$, and $\mathrm{Ag}$ [53]. The EDX results of ME-Ag NPs showed percentage relative composition of elements such as oxygen (O) $62.68 \%$, carbon (C) $37.05 \%$, and silver (Ag) $0.27 \%$. According to BET results, we found that the surface area was $220.43 \mathrm{~m}^{2} / \mathrm{g}$.

The FTIR technique of (a) pure matcha powder and (b) ME-Ag NPs was used to investigate functional groups of structure in the region between 4000 and $400 \mathrm{~cm}^{-1}$. FTIR analysis of pure matcha powder and ME-Ag NPs is shown in Fig. 2. According to the FTIR results, the characteristic peaks of pure matcha powder were observed at $3278.39 \mathrm{~cm}^{-1}$ (-OH stretching vibration), $2918.73 \mathrm{~cm}^{-1}(\mathrm{C}-\mathrm{H}$ stretching vibration), $1623.77 \mathrm{~cm}^{-1}$ (-OH bending and stretching vibrations), $1516.74 \mathrm{~cm}^{-1}(\mathrm{C}=\mathrm{O})$, $1444.42 \mathrm{~cm}^{-1}(\mathrm{C}=\mathrm{O}), 1366.32 \mathrm{~cm}^{-1}(\mathrm{C}=\mathrm{O}), 1232.29 \mathrm{~cm}^{-1}$ (-C-OH bending), $1143.58 \mathrm{~cm}^{-1}(\mathrm{C}=\mathrm{O})$, and $1016.3 \mathrm{~cm}^{-1}(\mathrm{C}-\mathrm{O}-\mathrm{C}$ vibrations $)$. The FTIR result was attributed to the (-)-Epigallocatechin-3-Gallate (EGCG) [54]. Furthermore, the characteristic peaks of ME-Ag NPs were observed at $3228.25 \mathrm{~cm}^{-1}(-\mathrm{OH}$ stretching vibration), $2927.41 \mathrm{~cm}^{-1}\left(\mathrm{C}-\mathrm{H}\right.$ stretching vibration), $1690.3 \mathrm{~cm}^{-1}$ (-OH bending and stretching vibrations), $1553.38 \mathrm{~cm}^{-1}(\mathrm{C}=\mathrm{O}), 1352.82 \mathrm{~cm}^{-1}(\mathrm{C}=\mathrm{O})$,
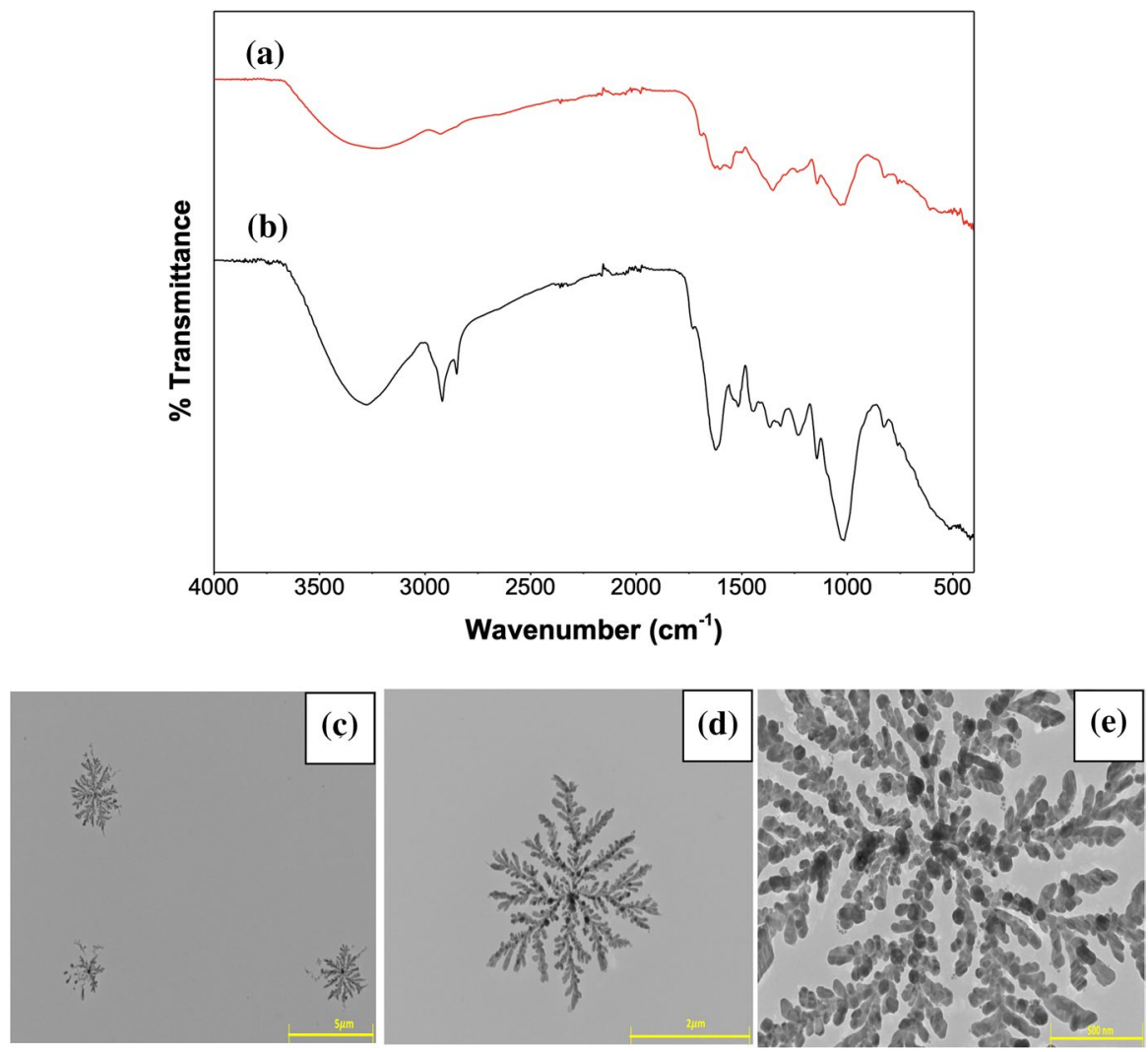

Fig. 2 FTIR spectra's of a pure matcha powder, and b ME-Ag NPs and TEM images of $\mathbf{c}$ ME-Ag NPs $\mathrm{x} 3 \mathrm{k}$, d ME-Ag NPs x10k, and e ME-Ag NPs x30k with different magnifications 
$1237.11 \mathrm{~cm}^{-1}$ (-C-OH bending), $1142.62 \mathrm{~cm}^{-1}(\mathrm{C}=\mathrm{O}), 1014.37 \mathrm{~cm}^{-1}(\mathrm{C}-\mathrm{O}-\mathrm{C}$ vibrations). These small shifts were due to the adsorption on the surface for $\mathrm{C}=\mathrm{O}$ [55]. Consequently, we assumed that EGCG had a significant effect on reduction of $\mathrm{Ag}^{+}$in NPs. TEM technique was used to characterize the of the surface of ME-Ag NPs. Figure 2 shows the TEM images of (c) ME-Ag NPs x3k, (d) ME-Ag NPs x10k, and (e) ME-Ag NPs x30k with different magnifications. According to TEM results, we found that the NPs had a hyperbranched unimolecular structure due to the contribution of the Ag nanoparticles around the branches of the polyphenolic dendrimer. Briefly, we observed that 2D hyperbranched Ag nanoparticles in the lateral size range of about 10-20 $\mathrm{nm}$ were synthesized via a self-assembly sonication process at room temperature for $30 \mathrm{~min}$ using of green reducing agent, leading to the fabrication of dendrimer-branched Ag nanoparticles [56]. Also, Ag nanoparticles were well separated in the structure. The results were compatible with our SEM results. We assumed that the polyphenolic dendrimer homogenously stabilizes silver nanoparticles by branching the nanostructure.

$\mathrm{X}$-ray diffraction (XRD) analysis of ME-Ag NPs was performed to calculate the crystalline size of Ag NPs in the structure. The XRD graph of the synthesized ME-Ag NPs is given in Fig. 3. Figure 3 shows that characteristic peaks were formed around $2 \theta=38.16^{\circ}, 41.22^{\circ}, 44.34^{\circ}, 56.38^{\circ}, 62.32^{\circ}, 64.52^{\circ}$, and $64.84^{\circ}$ corresponded to JCPDS file No.00-004-0783. We calculated the crystalline size of Ag NPs using different XRD mathematical models such as the Scherrer, modified Scherrer, and Williamson-Hall equations. The plot of (a) modified Scherrer and (b) Williamson-Hall of ME-Ag NPs is shown in Fig. 3. The crystalline sizes of NPs were found $5.21 \mathrm{~nm}, 15.98 \mathrm{~nm}$, and $15.74 \mathrm{~nm}$ by applying Scherrer, modified Scherrer, and Williamson-Hall formula, respectively (Fig. 3). These results were well coincide with the SEM and TEM results. Also, these results were in good agreement with a related study [57].

The synthesized ME-Ag NPs were characterized by AFM for three-dimensional morphology, topographical characterization, and agglomeration of Ag nanoparticles. The images were $5 \mu \mathrm{m} \times 5 \mu \mathrm{m}$. In Fig. 4, (a) AFM topographical image, (b) threedimensional morphology, and (c) cross section of image of ME-Ag NPs were found to be in line with the SEM and TEM, showing the sizes of the synthesized ME-Ag NPs as $15 \mathrm{~nm}$. The corresponding cross sections of the image was provided to show differences in height distributions between particles. According to AFM results, we observed that the ME-Ag NPs had a uniform size distribution and homogenous structure due to the polyphenolic network. Our results of AFM about surface topography of the synthesized ME-Ag NPs showed similarity to the results reported by many researchers concerning the surface characterization of $\mathrm{Ag}$ nanoparticles with uniform size distribution [58-61].

\section{Storage stability of the nanostructure}

Various previous studies have proven the structure, content, and morphological properties of nanoparticles effect thermal conductivity, rheological behavior, viscosity, and stability [62]. Regarding the morphology-dependent rheological 

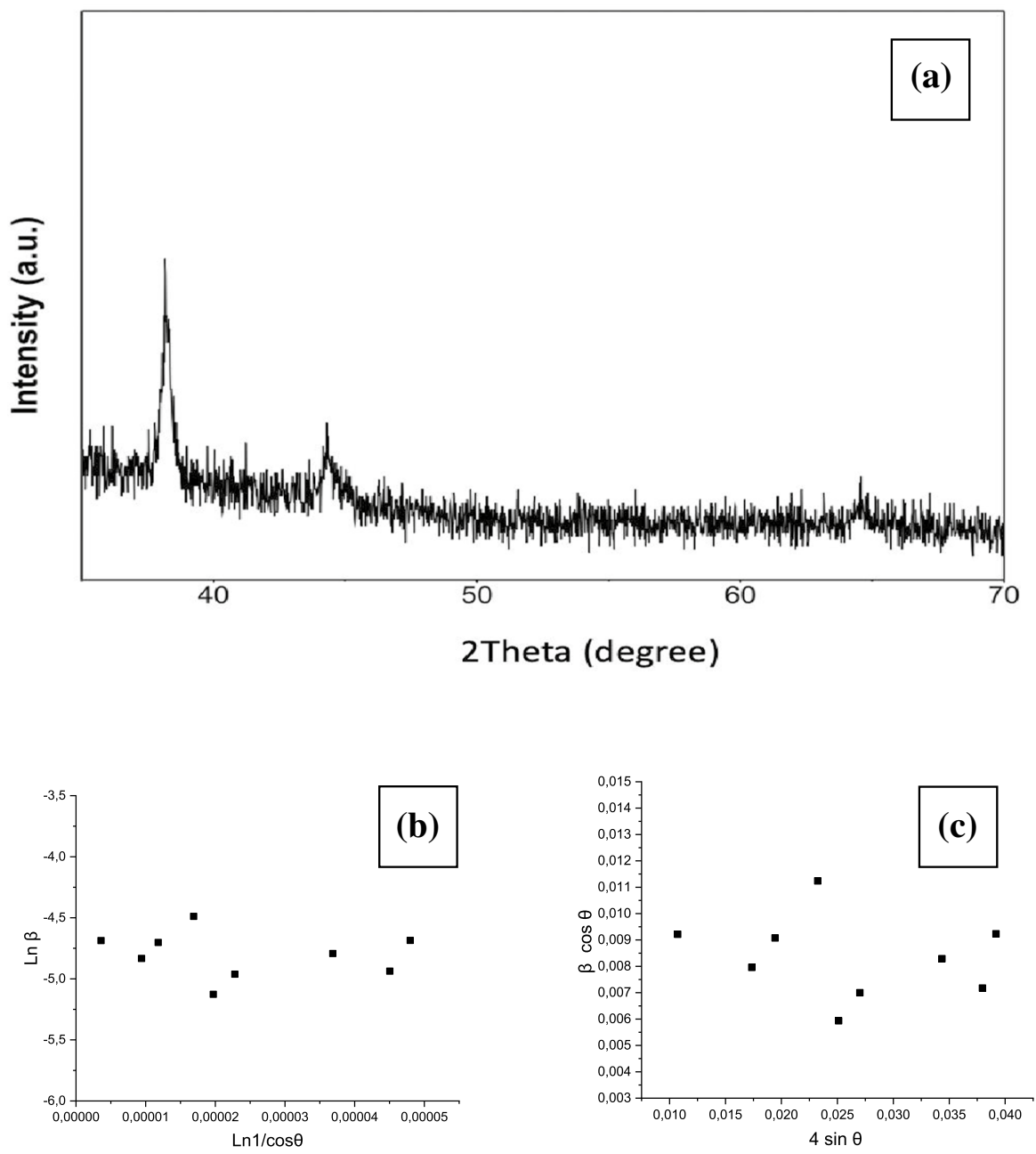

Fig. 3 a XRD result of the synthesized ME-Ag NPs, the plot of $\mathbf{b}$ modified Scherrer, and $\mathbf{c}$ WilliamsonHall of ME-Ag NPs

behavior and intrinsic viscosity measurement of nanofluids rich in Ag NPs, the lack of data in the literature is noteworthy. For this reason, we focused on the optimization of the viscosity of Ag NPs to have a long-term stability. The photographs of ME-Ag NPs after standing for 20 weeks (Fig. 5) showed that only a small amount of Ag NPs was precipitated at the bottom of the glass without obvious stratification, indicating their high storage stability. Furthermore, we found that Ag NPs had a high stable dispersion with small sizes in low concentrations and intrinsic viscosities of nanosystems. The storage stability of the hyperbranched unimolecular ME-Ag NPs was evaluated using rheological measurements. In Fig. 5, the intrinsic viscosity/time plot of the ME-Ag NPs, and the 

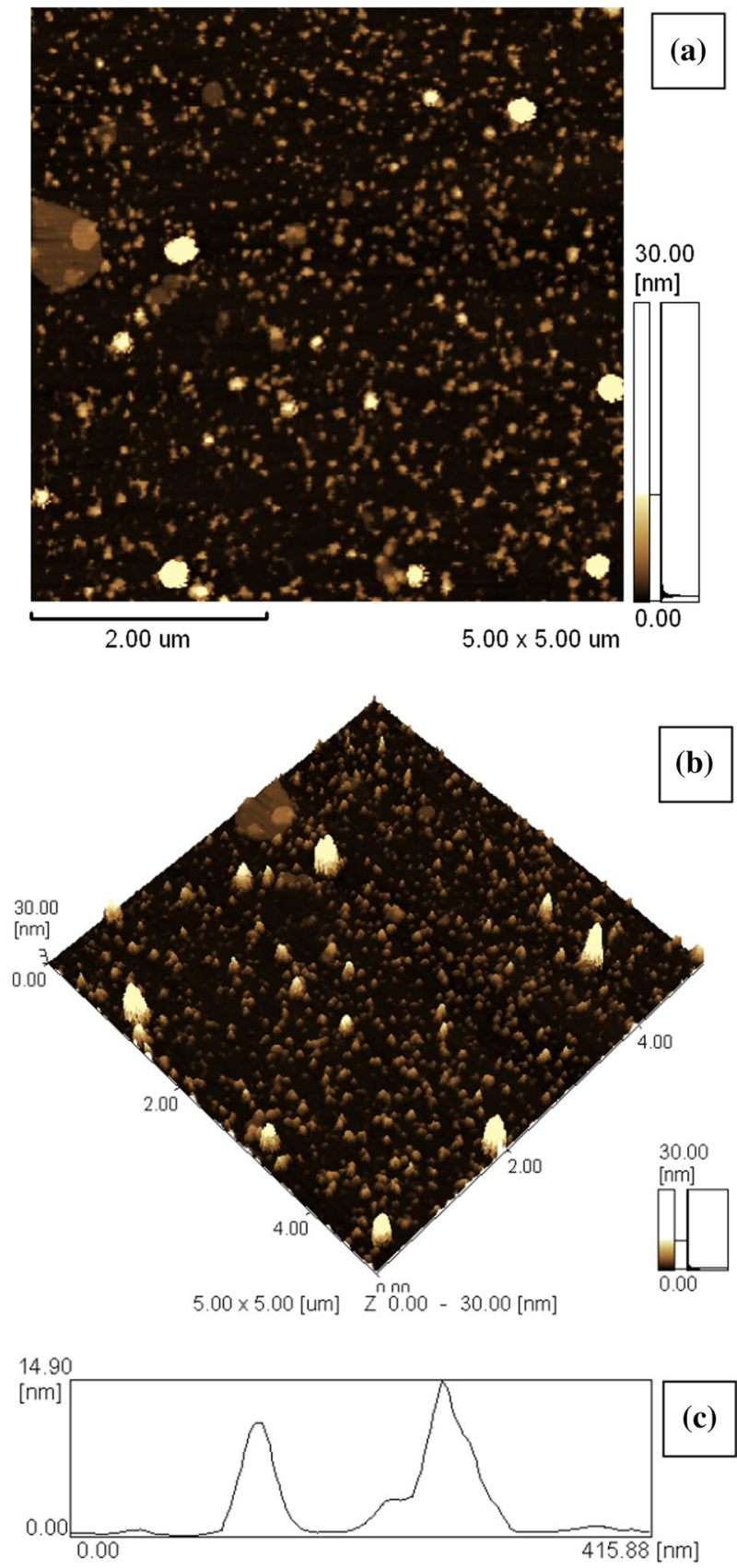

Fig. 4 a AFM topographical image, $\mathbf{b}$ three-dimensional morphology, and c cross-section of image of ME-Ag NPs 
(a)
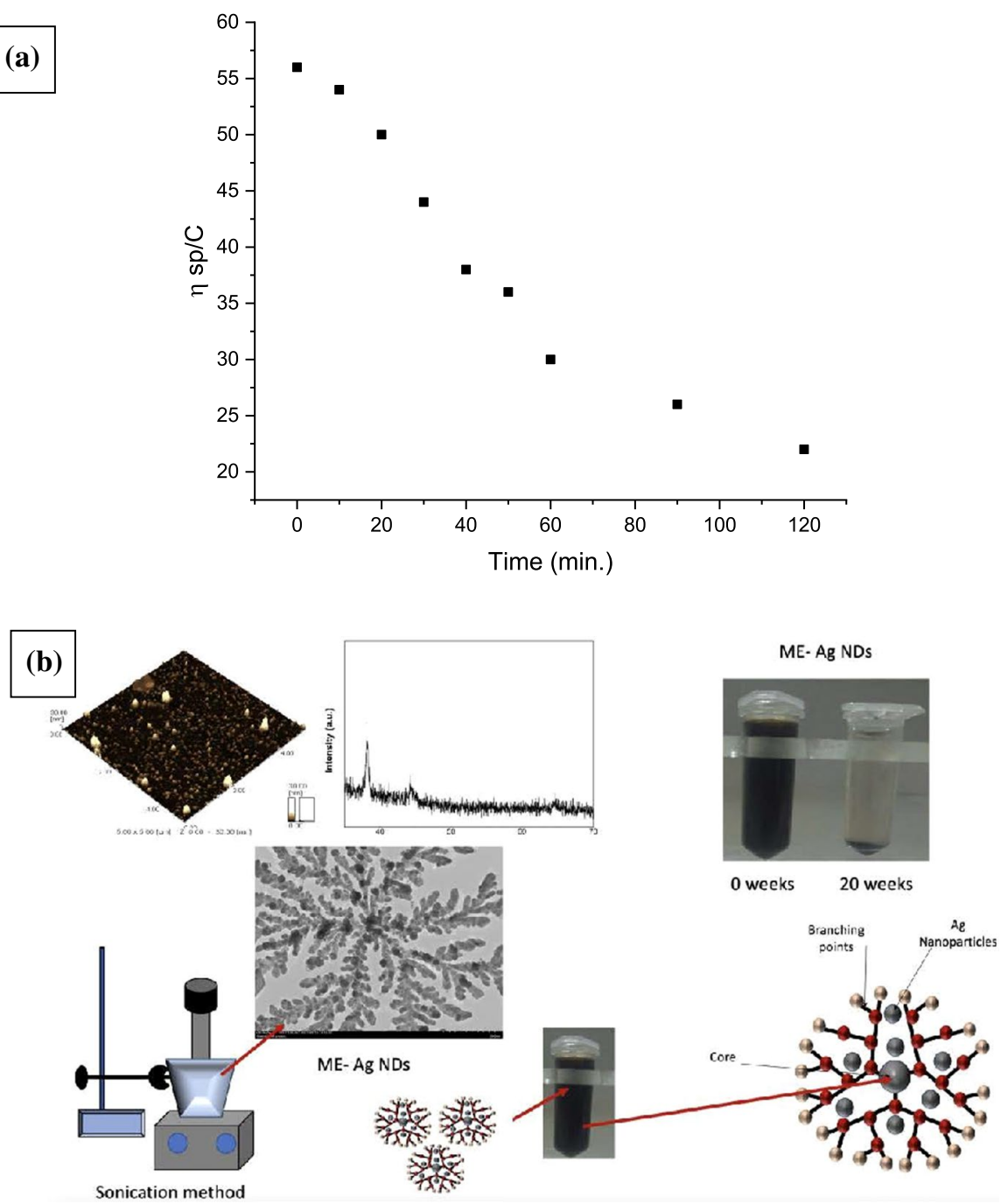

Fig. 5 a The intrinsic viscosity/time plot of the ME-Ag NPs, and $\mathbf{b}$ schematic image of stability of ME-Ag NPs

schematic image of stability of SLE- Ag NPs at different times were given. The storage stability of the synthesized ME-Ag NPs was monitored by checking its intrinsic viscosity at $25 \pm 0.1^{\circ} \mathrm{C}$. Also, the photograph of the ME-Ag NPs was taken over time (0-20 Week). Furthermore, the intrinsic viscosity difference of the ME-Ag NPs after 20 weeks of storage was found to be $34 \pm 1.0 \mathrm{dl} / \mathrm{g}$. These stability results exhibited an indication of its excellent storage stability with its photographs for the ME-Ag NPs. 


\section{Challenge test of the nanostructure}

Recently, Coronavirus (COVID-19) is a significant issue and related to nanosized virus so we should improve the antimicrobial activity of novel materials and to prevent the spread of these pathogenic problems using nanotechnological solutions. With this viewpoint, we developed the novel ME-Ag NPs and investigated the biological activity of the NPs to evaluate the preservative efficacy of ME-Ag NPs against pathogenic microorganisms such as Staphylococcus aureus (S. aureus), Pseudomonas aureginosa (P. aeruginosa), Escherichia coli (E. coli), Candida albicans (C. albicans), and Aspergillus brasilliensis (A. brasilliensis), which cause infectious disease. These microorganisms are known major problems and cause infections to people or animals in direct or indirect routes [63, 64]. We used the total aerobic mesophilic microorganism (ISO 21149) and mold-yeast (ISO 16212) (cosmetics-microbiology-evaluation of the antimicrobial protection of a cosmetic product) guidelines for the antimicrobial and antifungal activity of the synthesized ME-Ag NPs. The preservative efficacy of ME-Ag NPs against pathogenic microorganisms of ME-Ag NPs were given in Fig. 6. Concerning S. aureus, P. aeruginosa, E. coli, C. albicans, and A. brasilliensis, criterion A was satisfied for the synthesized ME-Ag NPs. To our knowledge, this report is the first study on preservative efficacy of the synthesized ME-Ag NPs against the $S$. aureus, P. aeruginosa, E. coli, C. albicans, and A. Brasilliensis for use in cosmetic preparations.

In previous studies, various reports were showed that plant extract-based nanoformulations had excellent biological activity against pathogenic microorganisms. In this study, our aim was to fabricate the biodegradable ME-Ag NPs without using any toxic reducing agents. The results showed that the synthesized Ag NPs had excellent antimicrobial and antifungal properties, and these results were appreciable from the comparison with the results of previous studies in the literature (Table 4).

\section{Colorimetric image sensing behavior of the nanostructure}

The smartphone-based ME-Ag NPs biosensor was developed for analysis of $\mathrm{H}_{2} \mathrm{O}_{2}$, isopropyl alcohol, ethanol, ethyl acetate, ammonia, ascorbic acid, dopamine hydrochloride, progesterone, and glucose. The analyte was deposited on the color region of ME-Ag NP-coated paper substrate and kept for 1 min to reach equilibrium. For color histogram analysis, the signal color intensity of ME-Ag NP-coated paper was calculated using the RGB color detector application in smartphone after addition of the target analyte. Furthermore, the limit of detection (LOD) was calculated using standard calibration curve with the response (S) (y)-axis and the $\log \mathrm{H}_{2} \mathrm{O}_{2}$ concentrations on the horizontal $(x)$-axis. The selectivity detection of ME-Ag NPs toward various analytes, including $\mathrm{H}_{2} \mathrm{O}_{2}$, isopropyl alcohol, ethanol, ethyl acetate, ammonia, ascorbic acid, dopamine hydrochloride, progesterone, and glucose was conducted in the nanostructure-coated paper image with a smartphone and naked eye. Our experimental methodology was based on the fine adjustment of the color change with amount of the target analyte through pipetting attempts on the ME-Ag 

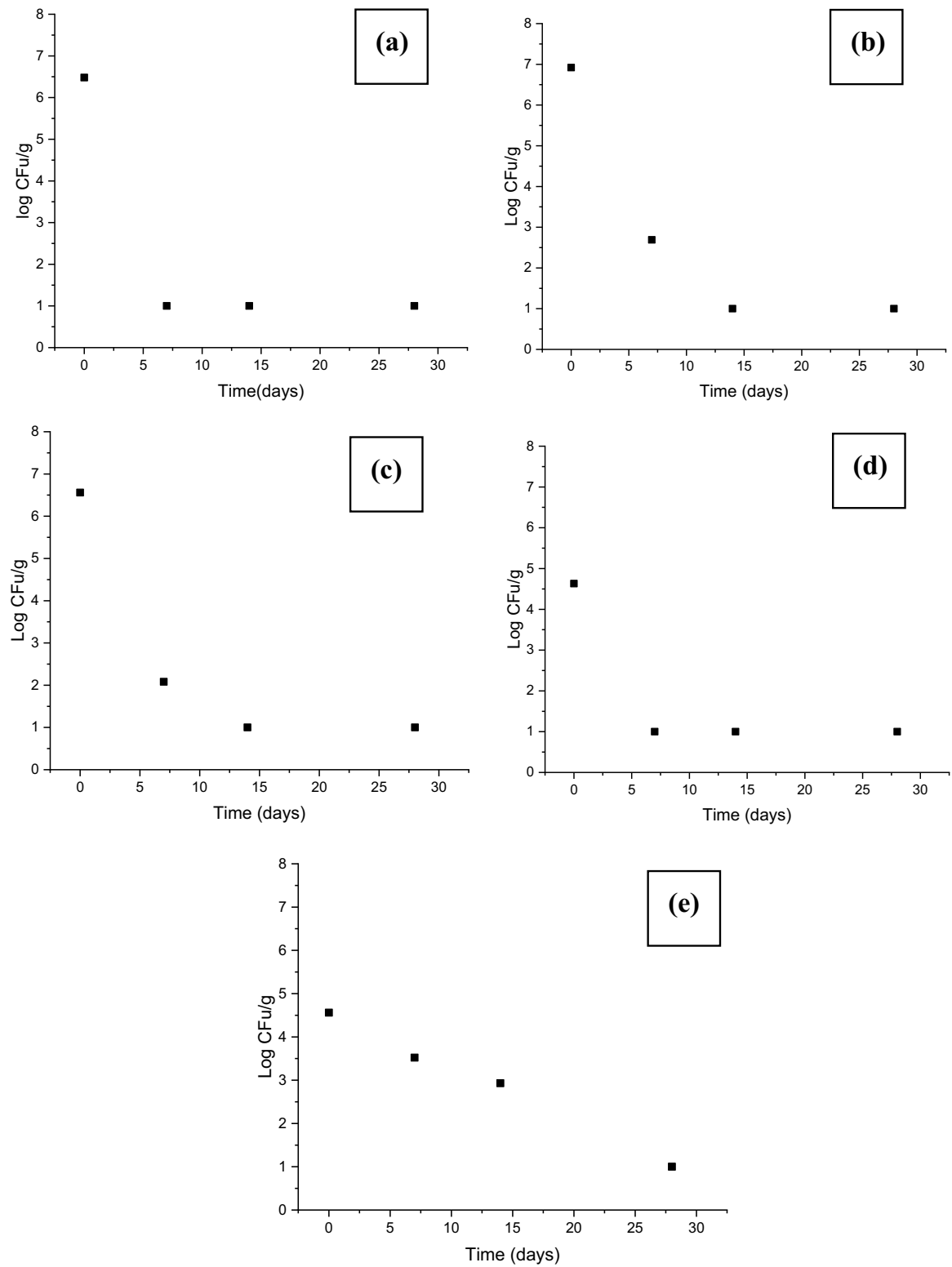

Fig. 6 The preservatives efficacy of the synthesized ME-Ag NPs against pathogenic microorganisms (a $S$. aureus, b P. aeruginosa, c E. coli, d C. albicans, and e A. brasilliensis)

NP $\left(10 \mu \mathrm{L}\right.$ NPs paste/ $\mathrm{cm}^{2}$ )-coated paper. All images (uncoated and coated paper) were captured from the top of the box using an autofocus mode in fixed viewing position between the detection smartphone camera and the biosensor (optimum distance: $8 \mathrm{~cm}$ ) [71]. 


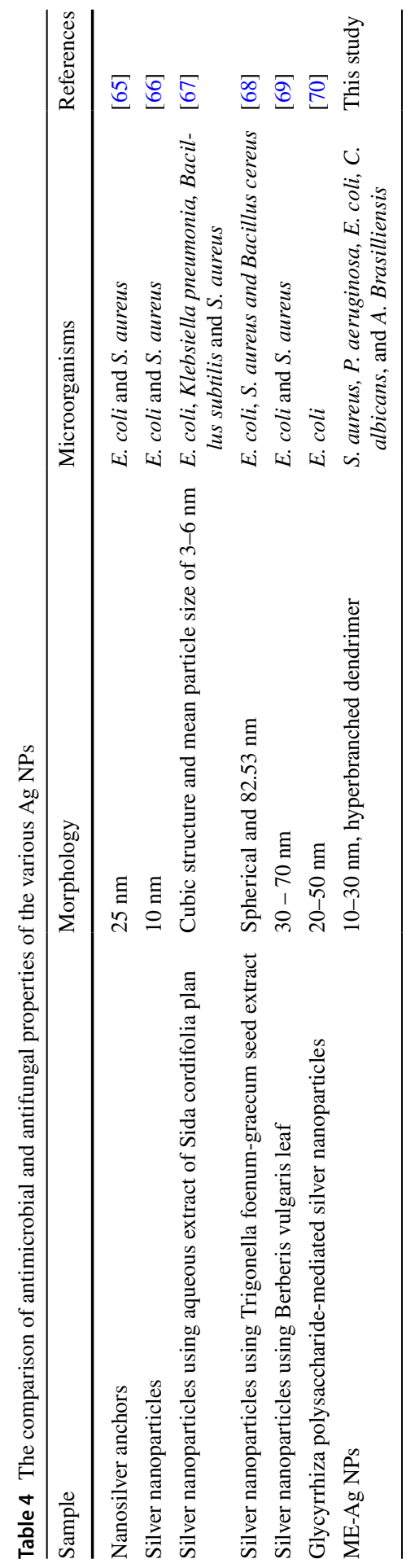


The analytical sensor performance of the smartphone-assisted biosensor for $\mathrm{H}_{2} \mathrm{O}_{2}$ detection was experimentally done under the optimal condition such as room temperature, sun light, and Casper VIA F20 model smartphone. The smartphone has $48+5+2+2$ MP quad rear camera, and the images of the samples were recorded in the continuum light source. With the camera supported by artificial intelligence, the samples were analyzed, and the best frames were captured [72].

In Fig. 7, the colorimetric analysis of smartphone camera-captured images of (a) ME-Ag NPs, and (b) ME-Ag NPs in the presence of $\mathrm{H}_{2} \mathrm{O}_{2}$, (c) ME-Ag NP-coated paper, (b) detection of $\mathrm{H}_{2} \mathrm{O}_{2}$ using the ME-Ag NP-coated paper biosensor were presented. In the smartphone-supported sensor study, we observed that the mean value of RGB increased due to the presence of $\mathrm{H}_{2} \mathrm{O}_{2}$, but there was no significant change in the presence of other analytes (isopropyl alcohol, ethanol, ethyl acetate, ammonia, ascorbic acid, dopamine hydrochloride, progesterone, and glucose was conducted in the nanostructure-coated paper image with a smartphone and naked eye.), according to RGB analysis. In the RGB method, the digital images of the NPs sensor collected by a smartphone camera were divided into three channels: red $(R)$, green $(G)$, and blue (B) due to the color histogram results obtained using ImageJ software and the corresponding chromatic values [7].

The small B value of NPs sensor showed the most remarkable response to $\mathrm{H}_{2} \mathrm{O}_{2}$. The $\mathrm{B}$ values were measured be $169,96,167,147,153,145,121,98,99,97$, and 100 for blank paper, ME-Ag NPs, ME-Ag NPs/ $\mathrm{H}_{2} \mathrm{O}_{2}$, ME-Ag NPs /isopropyl alcohol, ME-Ag NPs/ethanol, ME-Ag NPs/ethyl acetate, ME-Ag NPs/ammonia, ME-Ag NPs/ascorbic acid, ME-Ag NPs/dopamine hydrochloride, ME-Ag NPs/progesterone, and ME-Ag NPs/glucose, respectively.

In this study, we investigated the logarithmic correlation between the $\mathrm{H}_{2} \mathrm{O}_{2}$ concentration, and the response of the biosensor were determined from the calibration curve. The response (S) of sensor against logarithm of $\mathrm{H}_{2} \mathrm{O}_{2}$ concentration in the range from 0.05 to $20 \mu \mathrm{M}$ showed a linear relationship with a high correlation coefficient $\left(R^{2}\right)$ of 0.9957 . Based on experimental results of ME-Ag NP-coated paper biosensor, LOD was calculated using to the calibration curve and to be $0.82 \mu \mathrm{M}$ with a signal-to-noise ratio $(S / N=3)$ in the linear dynamic range of $0.05-20 \mu \mathrm{M}$ $\mathrm{H}_{2} \mathrm{O}_{2}$. We concluded that the developed smartphone-assisted method was successfully demonstrated for quantitative determination of $\mathrm{H}_{2} \mathrm{O}_{2}$.

The selectivity is known a significant characteristic of the sensor and the selectivity of sensor can be described using Euclidean distance $(\Delta \mathrm{E})$ values. The mathematical correlation between the calculated $\Delta E$ and analyte was shown in Fig. 7e, f. According to colorimetric image results, we observed that there was a significant change of intensity upon the addition of $\mathrm{H}_{2} \mathrm{O}_{2}$, while the presence of other analyte exhibited negligible variation in the color intensity. To investigate the effect of analyte on sensing performance, $\Delta E$ values were calculated to be 93.70, 75.74, 37.28, 62.51, and 66.79 for $\mathrm{H}_{2} \mathrm{O}_{2}$, isopropyl alcohol, ethanol, ethyl acetate, and ammonia, respectively. Also, we observed that the addition of $\mathrm{H}_{2} \mathrm{O}_{2}$ increased remarkable $\Delta E$ values of sensor and a color change was observed with the naked eye. Furthermore, the color changes for all isopropyl alcohol, ethanol, ethyl acetate, and ammonia analytes were negligible. The concentration of NPs is a major factor in the sensitive detection of the target analyte with low concentrations which determine the 
Fig. 7 The colorimetric analysis of smartphone camera-captured images of a ME-Ag NPs, and $\mathbf{b}$ ME-Ag NPs in the presence of $\mathrm{H}_{2} \mathrm{O}_{2}$, c ME-Ag NP-coated paper, d detection of $\mathrm{H}_{2} \mathrm{O}_{2}$ using the ME-Ag NPcoated paper biosensor, e selectivity analysis of biosensor, $\mathbf{f} \Delta \mathrm{E}-\log \mathrm{C}_{\mathrm{H} 2 \mathrm{O} 2}$ graph, $\mathbf{g}$ standard calibration of curve $\mathbf{h} \mathrm{Uv}-\mathrm{Vis}$ measurements of ME-Ag NPs/ $\mathrm{H}_{2} \mathrm{O}_{2}$, $\mathbf{i}$ the effect of concentration of ME-Ag NPs

economics sensor process. For this purpose, we analyzed the performance of the prepared ME-Ag NP-based sensor in different concentrations for detecting $\mathrm{H}_{2} \mathrm{O}_{2}$. Furthermore, we investigated the effect of concentrations of ME-Ag NPs in range of $0.5-10 \mu \mathrm{M}$ on the colorimetric detection of hydrogen peroxide and colorimetric measurement results using colorimetric Euclidean distance $(\Delta E)$ values of smartphone camera-captured images. We found that the ME-Ag NP-based sensor exhibited an extremely high sensitivity to $\mathrm{H}_{2} \mathrm{O}_{2}$ with a low concentration due to the strong interaction of $\mathrm{H}_{2} \mathrm{O}_{2}$ in the sensitive nanoprobe-coated filter paper. Additionaly, the minimum Ag NPs concentration was determined as $2.3 \mu \mathrm{M}$ for color change (Fig. 7i). According to our experimental results, the estimated detection mechanism of $\mathrm{H}_{2} \mathrm{O}_{2}$ is given in Eqs. 3.1-3.2.

$$
\begin{gathered}
\text { 2ME-AgNPs }+\mathrm{H}_{2} \mathrm{O}_{2} \leftrightarrow 2 \mathrm{ME}-\mathrm{AgNPs} \ldots \mathrm{OH}_{\mathrm{ad}} \\
\text { ME-AgNPs } \ldots \mathrm{OH}_{\mathrm{ad}}+\mathrm{e}^{-}+\mathrm{H}^{+} \leftrightarrow \text { ME-AgNPs }+\mathrm{H}_{2} \mathrm{O}
\end{gathered}
$$

A comparison of ME-Ag NP-based colorimetric $\mathrm{H}_{2} \mathrm{O}_{2}$ sensor with other previous results were given in Table 5. Our experimental results showed that the sensitive and low-cost ME-Ag NP-based sensor is suitable for colorimetric detection of $\mathrm{H}_{2} \mathrm{O}_{2}$.

Consequently, the ME-Ag NP-coated paper biosensor exhibited high selectivity for $\mathrm{H}_{2} \mathrm{O}_{2}$ and acted as a $\mathrm{H}_{2} \mathrm{O}_{2}$-selective nanoprobe-coated filter paper using the smartphone-assisted method with the RGB colorimetric approach. It was clear that the fabricated $\mathrm{H}_{2} \mathrm{O}_{2}$ sensing platform has a comparable LOD value than previous studies [2, 86, 87]. Using the smartphone-assisted color histogram analysis of image of ME-Ag NP-coated paper biosensor is a promising technique in sensor applications.

\section{Conclusion}

In this study, novel preservative nanostructure containing ME-Ag NPs enriched with polyphenol were prepared using a green and one-step sonochemical method via a self-assembly process. The antimicrobial and antifungal properties of the novel nanostructure was investigated against pathogenic microorganisms such as S. aureus, P. aeruginosa, E. coli, C. albicans, and A. brasilliensis. Characterization results showed that the synthesized ME-Ag NPs had a hyperbranched unimolecular, narrow size distribution $(10 \mathrm{~nm}$ to $30 \mathrm{~nm})$, and large surface area $\left(220.43 \mathrm{~m}^{2} / \mathrm{g}\right)$. Furthermore, naked eye and colorimetric measurements confirmed the significant color change for the sensing $\mathrm{H}_{2} \mathrm{O}_{2}$ using the smartphone-based ME-Ag NPs biosensors with the LOD of $0.82 \mu \mathrm{M}$, thereby it was found to be a low-cost, portable, and selective sensor for the determination of $\mathrm{H}_{2} \mathrm{O}_{2}$ using the color histogram analysis. 
(a)

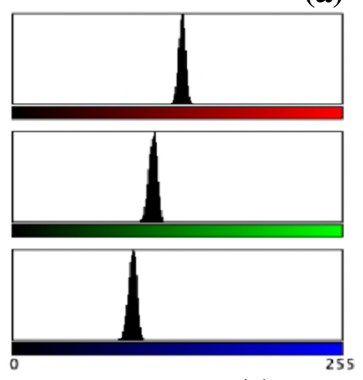

(c) (b)

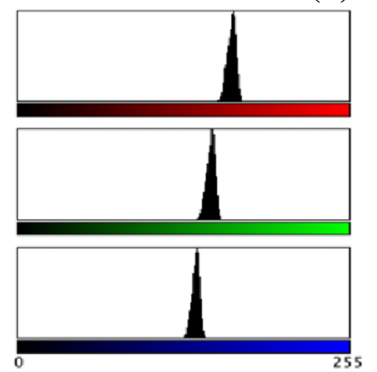

(d)

(e)
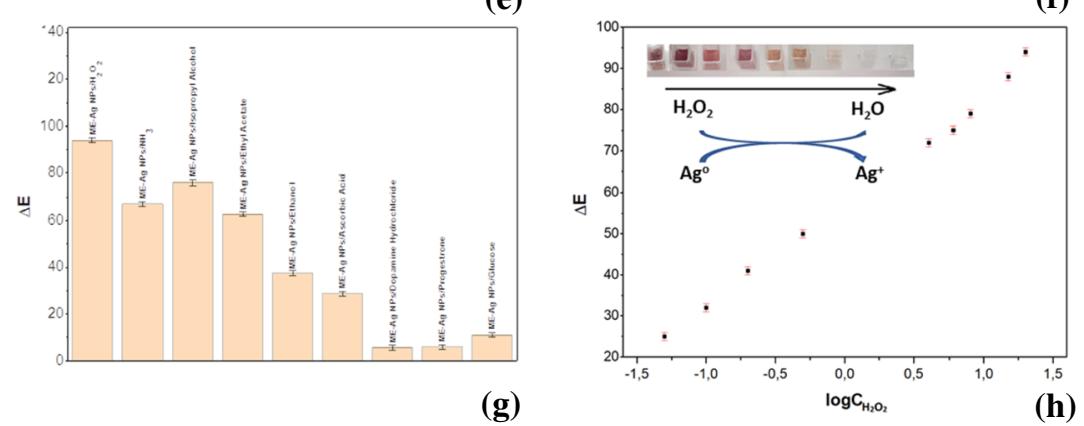

(g)
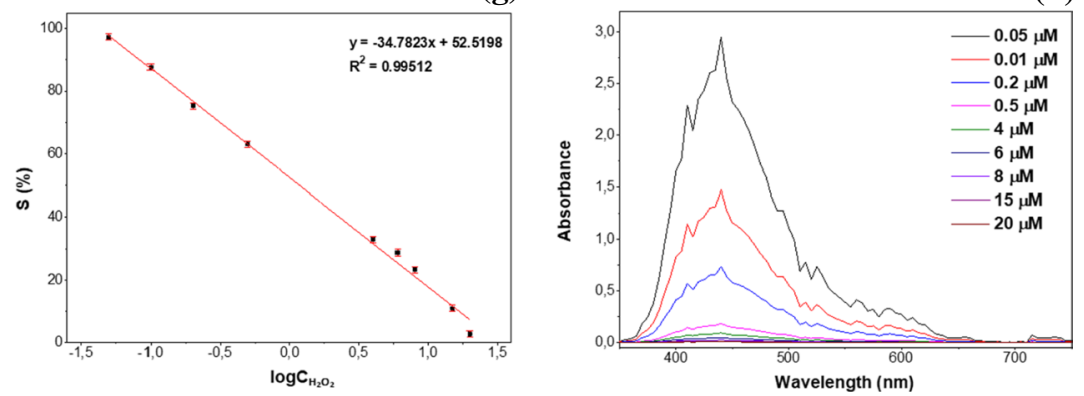

(i)

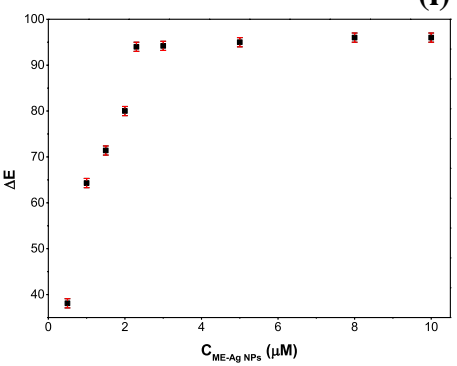


Table 5 The comparison of electrochemical results of the various sensors for the detection of $\mathrm{H}_{2} \mathrm{O}_{2}$

\begin{tabular}{|c|c|c|c|}
\hline Sample & $\begin{array}{l}\text { Limit of detection } \\
\text { (LOD) }\end{array}$ & Linear range & References \\
\hline Gold nanorods & $0.067 \mu \mathrm{M}$ & $0.20-30.00 \mu \mathrm{M}$ & [73] \\
\hline Ag colloidal nanoparticles & $0.2656 \mathrm{ppm}$ & $0-240 \mathrm{ppm}$ & [74] \\
\hline $\begin{array}{l}\text { Magnetic ternary nanocomposite hemin- }-\mathrm{Fe}_{3} \mathrm{O}_{4} \\
\text { polypyrrole }\end{array}$ & $0.18 \mu \mathrm{M}$ & $0.2-100 \mu \mathrm{M}$ & [75] \\
\hline $\begin{array}{l}\mathrm{Ru} \\
\mathrm{V} 2 \mathrm{O} 4 \text { nanowires }\end{array}$ & $0.788 \mu \mathrm{M}$ & $1-500 \mu \mathrm{M}$ & [76] \\
\hline MoOx quantum dots & $0.175 \mu \mathrm{mol} / \mathrm{L}$ & $1-20 \mu \mathrm{mol} / \mathrm{L}$ & {$[77]$} \\
\hline $\begin{array}{l}\text { Palygorskite } \\
\mathrm{Co}_{3} \mathrm{O}_{4} \\
\text { nanocomposites }\end{array}$ & $0.70 \mu \mathrm{M}$ & $0-80 \mu \mathrm{M}$ & [78] \\
\hline $\begin{array}{l}\text { Graphene oxide and } \mathrm{CuO} \text { double quantum dot com- } \\
\text { posites (GOQD-q-CuO) }\end{array}$ & $0.5 \mu \mathrm{M}$ & $2.5-200 \mu \mathrm{M}$ & [79] \\
\hline $\mathrm{CeO}_{2} \mathrm{NPs}$ & $0.5 \mu \mathrm{M}$ & $0.6-1.5 \mu \mathrm{M}$ & [80] \\
\hline Nickel metal-organic framework 2D nanosheets & $8 \mathrm{nM}$ & $0.04-160 \mu \mathrm{M}$ & [81] \\
\hline $\begin{array}{l}\mathrm{Au} \\
\text { CuxOS yolk-shell nanomaterials }\end{array}$ & $0.03 \mu \mathrm{M}$ & $0.1-32 \mu \mathrm{M}$ & [82] \\
\hline FePt-Au ternary metallic hybrid nanaoparticles & $12.33 \mu \mathrm{M}$ & $20-700 \mu \mathrm{M}$ & [83] \\
\hline Porphyrin modified $\mathrm{ZnFe}_{2} \mathrm{O}_{4} /$ reduced graphene oxide & $0.54 \mu \mathrm{M}$ & $0.7-30 \mu \mathrm{M}$ & [84] \\
\hline $\begin{array}{l}\text { Composites of } \mathrm{Fe}_{3} \mathrm{O}_{4} \text { modified by sodium lignosul- } \\
\text { fonate and copper peroxide }\left(\mathrm{Fe}_{3} \mathrm{O}_{4}\right. \\
\text { CP) }\end{array}$ & $0.11 \mu \mathrm{M}$ & $0.2-300 \mu \mathrm{M}$ & [85] \\
\hline ME-Ag NPs & $0.82 \mu \mathrm{M}$ & $0.05-20 \mu \mathrm{M}$ & This study \\
\hline
\end{tabular}

We concluded that the challenge test proved that it had an excellent antibacterial and antifungal activity with criteria A data in cosmetic applications.

Acknowledgements This work was supported by Scientific Research Projects Coordination Unit of Istanbul University-Cerrahpasa. Project number 32438.

\section{Declarations}

Conflict of interest The authors declare no conflict of interests.

\section{References}

1. Yuvashree S, Balavijayalakshmi J (2019) Graphene based nanocomposites for electrochemical detection of H2o2. In: Materials Today: Proceedings. Elsevier Ltd, pp 1740-1745

2. Fiore L, Mazzaracchio V, Galloni P et al (2021) A paper-based electrochemical sensor for $\mathrm{H} 2 \mathrm{O} 2$ detection in aerosol phase: measure of $\mathrm{H} 2 \mathrm{O} 2$ nebulized by a reconverted ultrasonic aroma diffuser as a case of study. Microchem J 166:106249. https://doi.org/10.1016/j.microc.2021.106249

3. Zhang W, Niu X, Li X et al (2018) A smartphone-integrated ready-to-use paper-based sensor with mesoporous carbon-dispersed Pd nanoparticles as a highly active peroxidase mimic for $\mathrm{H} 2 \mathrm{O} 2$ detection. Sens Actuators B Chem 265:412-420. https://doi.org/10.1016/j.snb.2018.03.082 
4. Luo W, Deng J, He J et al (2021) A smartphone-based multi-wavelength photometer for on-site detection of the liquid colorimetric assays for clinical biochemical analyses. Sens Actuators B Chem 329:129266. https://doi.org/10.1016/J.SNB.2020.129266

5. Xing Y, Zhu Q, Zhou X, Qi P (2020) A dual-functional smartphone-based sensor for colorimetric and chemiluminescent detection: a case study for fluoride concentration mapping. Sens Actuators B Chem 319:128254. https://doi.org/10.1016/j.snb.2020.128254

6. Shiferaw HM (2021) Measuring traffic induced ground vibration using smartphone sensors for a first hand structural health monitoring. Sci African 11:e00703. https://doi.org/10.1016/j.sciaf.2021. e00703

7. Purohit B, Kumar A, Mahato K, Chandra P (2020) Smartphone-assisted personalized diagnostic devices and wearable sensors. Curr Opin Biomed Eng 13:42-50

8. Fan Y, Li J, Guo Y et al (2021) Digital image colorimetry on smartphone for chemical analysis: a review. Meas J Int Meas Confed 171:108829. https://doi.org/10.1016/j.measurement.2020.108829

9. Jin R, Wang F, Li Q et al (2021) Construction of multienzyme-hydrogel sensor with smartphone detector for on-site monitoring of organophosphorus pesticide. Sens ACTUATORS B Chem 327:128922. https://doi.org/10.1016/j.snb.2020.128922

10. $\mathrm{Xu} \mathrm{W,} \mathrm{Lu} \mathrm{S,} \mathrm{Chen} \mathrm{Y} \mathrm{et} \mathrm{al} \mathrm{(2015)} \mathrm{Simultaneous} \mathrm{color} \mathrm{sensing} \mathrm{of} \mathrm{O2} \mathrm{and} \mathrm{pH} \mathrm{using} \mathrm{a} \mathrm{smartphone.}$ Sens Actuators B Chem 220:326-330. https://doi.org/10.1016/j.snb.2015.05.088

11. Zhou Z, Li Y, Su W et al (2019) A dual-signal colorimetric and near-infrared fluorescence probe for the detection of exogenous and endogenous hydrogen peroxide in living cells. Sens Actuators B Chem 280:120-128. https://doi.org/10.1016/J.SNB.2018.09.126

12. Cong C, Bian K, Zhang X et al (2020) Sensitive measurement of tumor markers somatostatin receptors using an octreotide-directed Pt nano-flakes driven electrochemical sensor. Talanta 208:120286. https://doi.org/10.1016/J.TALANTA.2019.120286

13. Li J, Jiang M, Su M et al (2021) Stretchable and transparent electrochemical sensor based on nanostructured $\mathrm{Au}$ on carbon nanotube networks for real-time analysis of $\mathrm{H} 2 \mathrm{O} 2$ release from cells. Anal Chem 93:6723-6730. https://doi.org/10.1021/acs.analchem.1c00336

14. Xie J, Cheng D, Zhou Z et al (2020) Hydrogen peroxide sensing in body fluids and tumor cells via in situ produced redox couples on two-dimensional holey $\mathrm{CuCo} 2 \mathrm{O} 4$ nanosheets. Microchimica Acta. https://doi.org/10.1007/s00604-020-04389-2

15. Ma Z, Sun Y, Xie J et al (2020) Facile preparation of MnO2 quantum dots with enhanced fluorescence via microenvironment engineering with the assistance of some reductive biomolecules. ACS Appl Mater Interfaces 12:15919-15927. https://doi.org/10.1021/acsami.0c00917

16. Yao J, Wu T, Sun Y et al (2019) A novel biomimetic nanoenzyme based on ferrocene derivative polymer NPs coated with polydopamine. Talanta 195:265-271. https://doi.org/10.1016/J.TALAN TA.2018.11.069

17. Wu Q, Chen H, Fang A et al (2017) Universal multifunctional nanoplatform based on target-induced in situ promoting Au seeds growth to quench fluorescence of upconversion nanoparticles. ACS Sens 2:1805-1813. https://doi.org/10.1021/acssensors.7b00616

18. Zhao H, Ma H, Li X et al (2021) Nanocomposite of halloysite nanotubes/multi-walled carbon nanotubes for methyl parathion electrochemical sensor application. Appl Clay Sci 200:105907. https:// doi.org/10.1016/J.CLAY.2020.105907

19. Song J, Huang M, Lin X et al (2022) Novel Fe-based metal-organic framework (MOF) modified carbon nanofiber as a highly selective and sensitive electrochemical sensor for tetracycline detection. Chem Eng J 427:130913. https://doi.org/10.1016/J.CEJ.2021.130913

20. Karimi-Maleh H, Yola ML, Atar N et al (2021) A novel detection method for organophosphorus insecticide fenamiphos: molecularly imprinted electrochemical sensor based on core-shell Co3O4@ MOF-74 nanocomposite. J Colloid Interface Sci 592:174-185. https://doi.org/10.1016/J.JCIS.2021. 02.066

21. Ding C, Liu X, Yang S (2021) The value of infectious disease modeling and trend assessment: a public health perspective. Expert Rev Anti Infect Ther. https://doi.org/10.1080/14787210.2021. 1882850

22. Lima WG, Brito JCM, Cruz Nizer WS (2021) Bee products as a source of promising therapeutic and chemoprophylaxis strategies against COVID-19 (SARS-CoV-2). Phyther Res 35:743-750. https:// doi.org/10.1002/ptr.6872

23. Rangayasami A, Kannan K, Murugesan S et al (2021) Influence of nanotechnology to combat against COVID-19 for global health emergency: a review. Sensors Int 2:100079. https://doi.org/10. 1016/j.sintl.2020.100079 
24. Ahmed SA, Al- Mousawi AJ (2021) Preparation of lysozyme enzyme coated with chitosan nanoparticles. Plant Arch 21:1079-1087. https://doi.org/10.51470/plantarchives.2021.v21.s1.169

25. Hasan KMF, Horváth PG, Alpár T (2021) Lignocellulosic fiber cement compatibility: a State of the Art Review. J Nat Fibers

26. Nouri M, Baghaee-Ravari S, Emadzadeh B (2021) Nano-emulsified savory and thyme formulation show limited efficacy to suppress Pectobacterium carotovorum subsp. carotovorum compared with pure oil. Ind Crops Prod 161:113216. https://doi.org/10.1016/j.indcrop.2020.113216

27. Tegenaw A, Sorial GA, Sahle-Demessie E (2021) Effect of colloid-size copper-based pesticides and wood-preservatives against microbial activities of Gram-positive Bacillus species using five-day biochemical oxygen demand test. J Environ Sci (China) 105:71-80. https://doi.org/10.1016/j.jes. 2020.12.037

28. Sun Q, Li J, Le T (2018) Zinc oxide nanoparticle as a novel class of antifungal agents: current advances and future perspectives. J Agric Food Chem 66:11209-11220. https://doi.org/10.1021/acs. jafc. $8 \mathrm{~b} 03210$

29. Liu J, Wu D, Zhu N et al (2021) Antibacterial mechanisms and applications of metal-organic frameworks and their derived nanomaterials. Trends Food Sci Technol 109:413-434

30. Balasubramaniam B, Prateek RS et al (2021) Antibacterial and antiviral functional materials: chemistry and biological activity toward tackling COVID-19-like pandemics. ACS Pharmacol Transl Sci 2021:54

31. Sandhya PK, Jose J, Sreekala MS et al (2018) Reduced graphene oxide and ZnO decorated graphene for biomedical applications. Ceram Int 44:15092-15098. https://doi.org/10.1016/j.ceramint.2018. 05.143

32. Rajakumari R, Volova T, Oluwafemi OS et al (2020) Nano formulated proanthocyanidins as an effective wound healing component. Mater Sci Eng C 106:110056. https://doi.org/10.1016/j.msec. 2019.110056

33. Khatami M, Alijani HQ, Heli H, Sharifi I (2018) Rectangular shaped zinc oxide nanoparticles: green synthesis by Stevia and its biomedical efficiency. Ceram Int 44:15596-15602. https://doi.org/ 10.1016/j.ceramint.2018.05.224

34. Rousse C, Josse J, Mancier V et al (2016) Synthesis of copper-silver bimetallic nanopowders for a biomedical approach; study of their antibacterial properties. RSC Adv 6:50933-50940. https://doi. org/10.1039/c6ra07002g

35. Mohan S, Oluwafemi OS, Kalarikkal N, Thomas S (2015) Antibacterial and sensing properties of dextrose reduced starch - capped silver nanoparticles synthesised via a completely green method. In: Materials Today: Proceedings. Elsevier Ltd, pp 3943-3949

36. Yan K, Xu F, Wei W et al (2021) Electrochemical synthesis of chitosan/silver nanoparticles multilayer hydrogel coating with $\mathrm{pH}$-dependent controlled release capability and antibacterial property. Colloids Surf B Biointerf 202:111711. https://doi.org/10.1016/J.COLSURFB.2021.111711

37. Choudhary M, Chhabra P, Tyagi A, Singh H (2021) Scar free healing of full thickness diabetic wounds: a unique combination of silver nanoparticles as antimicrobial agent, calcium alginate nanoparticles as hemostatic agent, fresh blood as nutrient/growth factor supplier and chitosan as base matrix. Int J Biol Macromol 178:41-52. https://doi.org/10.1016/J.IJBIOMAC.2021.02.133

38. Philip P, Jose T, Prakash J, Cherian SK (2021) Surface plasmon resonance-enhanced bathochromic-shifted photoluminescent properties of pure and structurally modified electrospun poly(methyl methacrylate) (PMMA) nanofibers incorporated with green-synthesized silver nanoparticles graphic abstract keywords electrospinning PMMA AgNPs photoluminescence. J Electron Mater 50:4834-4849

39. Alibwaini YA, Hemeda OM, El-Shater R et al (2021) Synthesis, characterizations, optical and photoluminescence properties of polymer blend PVA/PEG films doped eosin Y (EY) dye. Opt Mater (Amst) 111:110600. https://doi.org/10.1016/J.OPTMAT.2020.110600

40. Nguyen N, Le CH (2021) Synthesis of PVA encapsulated silver nanoparticles as a drug delivery system for doxorubicin and curcumin. Int J High School Res. https://doi.org/10.36838/v3i3.9

41. Abd El-Kader MFH, Elabbasy MT, Ahmed MK, Menazea AA (2021) Structural, morphological features, and antibacterial behavior of PVA/PVP polymeric blends doped with silver nanoparticles via pulsed laser ablation. J Mater Res Technol 13:291-300. https://doi.org/10.1016/J.JMRT.2021. 04.055

42. Calamak S, Ermis M (2021) In situ silver nanoparticle synthesis on 3D-printed polylactic acid scaffolds for biomedical applications. J Mater Res. https://doi.org/10.1557/s43578-020-00064-7 
43. Burridge K, Johnston J, Borrmann T (2011) Silver nanoparticle-clay composites. J Mater Chem. https://doi.org/10.1039/c0jm02702b

44. Nazeruddin GM, Prasad NR, Waghmare SR et al (2014) Extracellular biosynthesis of silver nanoparticle using Azadirachta indica leaf extract and its anti-microbial activity. J Alloys Compd 583:272-277. https://doi.org/10.1016/J.JALLCOM.2013.07.111

45. Niu L, Coleman JN, Zhang H et al (2016) Production of two-dimensional nanomaterials via liquidbased direct exfoliation. Small 12:272-293. https://doi.org/10.1002/smll.201502207

46. Le Ba T, Mahian O, Wongwises S, Szilágyi IM (2020) Review on the recent progress in the preparation and stability of graphene-based nanofluids. J Therm Anal Calorim 142:1145-1172

47. Khan M, Mishra A, Shukla J, Sharma P (2019) X-ray analysis of BaTiO3 ceramics by WilliamsonHall and size strain plot methods. In: AIP Conference Proceedings. American Institute of Physics Inc., p 020138

48. Emil E, Gürmen S (2018) Estimation of yttrium oxide microstructural parameters using the Williamson-Hall analysis. Mater Sci Technol 34:1549-1557. https://doi.org/10.1080/02670836.2018. 1490857

49. Liu X, Chen Z, Gao R et al (2021) Portable quantitative detection of Fe3+ by integrating a smartphone with colorimetric responses of a rhodamine-functionalized polyacrylamide hydrogel chemosensor. Sens Actuators B Chem 340:129958. https://doi.org/10.1016/j.snb.2021.129958

50. Engel L, Benito-Altamirano I, Tarantik KR et al (2021) Printed sensor labels for colorimetric detection of ammonia, formaldehyde and hydrogen sulfide from the ambient air. Sens Actuators B Chem 330:129281. https://doi.org/10.1016/j.snb.2020.129281

51. Asghar MA, Zahir E, Shahid SM et al (2018) Iron, copper and silver nanoparticles: green synthesis using green and black tea leaves extracts and evaluation of antibacterial, antifungal and aflatoxin B1 adsorption activity. LWT Food Sci Technol 90:98-107. https://doi.org/10.1016/j.lwt.2017.12.009

52. Luo Q, Zhang J-R, Li H-B et al (2020) Green extraction of antioxidant polyphenols from green tea (Camellia sinensis). Antioxidants 9:785. https://doi.org/10.3390/antiox9090785

53. Femi-Adepoju AG, Dada AO, Otun KO et al (2019) Green synthesis of silver nanoparticles using terrestrial fern (Gleichenia Pectinata (Willd.) C. Presl.) characterization and antimicrobial studies. Heliyon 5:e01543. https://doi.org/10.1016/j.heliyon.2019.e01543

54. Narmada IB, Sarasati A, Wicaksono S et al (2020) Phytochemical screening, antioxidant activity, functional groups and chemical element characterization analysis of (-)-epigallocatechin-3-gallate (EGCG) in East Javanese Green Tea methanolic extract: an experimental in vitro study. Syst Rev Pharm. https://doi.org/10.31838/srp.2020.5.68

55. AbuDalo MA, Al-Mheidat IR, Al-Shurafat AW et al (2019) Synthesis of silver nanoparticles using a modified Tollens' method in conjunction with phytochemicals and assessment of their antimicrobial activity. PeerJ 2019:e6413. https://doi.org/10.7717/peerj.6413

56. Luo Y, Sun X (2007) Rapid, single-step preparation of dendrimer-protected silver nanoparticles through a microwave-based thermal process. Mater Lett 61:1622-1624. https://doi.org/10.1016/j. matlet.2006.07.087

57. Bindhu MR, Umadevi M, Esmail GA et al (2020) Green synthesis and characterization of silver nanoparticles from Moringa oleifera flower and assessment of antimicrobial and sensing properties. J Photochem Photobiol B Biol 205:111836. https://doi.org/10.1016/j.jphotobiol.2020.111836

58. Harisha KS, Parushuram N, Ranjana R et al (2020) Characterization and antibacterial properties of biogenic spherical silver nanoparticles. Mater Today Proc. https://doi.org/10.1016/j.matpr.2020.09. 654

59. Samide A, Iacobescu GE, Tutunaru B, Iordache S (2021) Silver nanoparticles/polyvinyl alcohol film: studies of thermal characterization, AFM and corrosion protection by electrodeposition on 304L stainless steel. J Therm Anal Calorim. https://doi.org/10.1007/s10973-020-10444-0

60. Nayak S, Bhat MP, Udayashankar AC et al (2020) Biosynthesis and characterization of Dillenia indica-mediated silver nanoparticles and their biological activity. Appl Organomet Chem 34:e5567. https://doi.org/10.1002/aoc.5567

61. Singh P, Pandit S, Mokkapati V et al (2020) A sustainable approach for the green synthesis of silver nanoparticles from Solibacillus isronensis sp. and their application in biofilm inhibition. Molecules 25:2783. https://doi.org/10.3390/molecules 25122783

62. Bao Z, Bing N, Zhu X et al (2021) Ti3C2Tx MXene contained nanofluids with high thermal conductivity, super colloidal stability and low viscosity. Chem Eng J 406:126390. https://doi.org/10. 1016/J.CEJ.2020.126390 
63. Amaral LFB, Camilo NS, Pereda MDCV et al (2011) Evaluation of antimicrobial effectiveness of C-8 xylitol monoester as an alternative preservative for cosmetic products. Int J Cosmet Sci 33:391397. https://doi.org/10.1111/j.1468-2494.2010.00633.x

64. Chorilli M, Leonardi GR, Nunes Salgado HR, Scarpa MV (2011) Evaluation of preservative effectiveness of liquid crystalline systems with retynil palmitate by the challenge test and d-value. J AOAC Int 94:118-127. https://doi.org/10.1093/jaoac/94.1.118

65. Priyadarshini S, Sonsudin F, Mainal A et al (2021) Phytosynthesis of biohybrid nano-silver anchors enhanced size dependent photocatalytic, antibacterial, anticancer properties and cytocompatibility. Process Biochem 101:59-71. https://doi.org/10.1016/j.procbio.2020.11.008

66. Abdulqahar FW, Almuhamady AK, Al Taee MA (2021) Characterization and antibacterial activity of silver nanoparticles biosynthesized by using aquatic weeds extracts. Environ Nanotechnol Monit Manag 15:100406. https://doi.org/10.1016/j.enmm.2020.100406

67. Pallela PNVK, Ummey S, Ruddaraju LK et al (2018) Ultra Small, mono dispersed green synthesized silver nanoparticles using aqueous extract of Sida cordifolia plant and investigation of antibacterial activity. Microb Pathog 124:63-69. https://doi.org/10.1016/j.micpath.2018.08.026

68. Awad MA, Awatif A, Hendi A, Ortashi KM et al (2021) Biogenic synthesis of silver nanoparticles using Trigonella foenum-graecum seed extract: Characterization, photocatalytic and antibacterial activities. Sens Actuators A Phys 323:112670. https://doi.org/10.1016/j.sna.2021.112670

69. Behravan M, Hossein Panahi A, Naghizadeh A et al (2019) Facile green synthesis of silver nanoparticles using Berberis vulgaris leaf and root aqueous extract and its antibacterial activity. Int J Biol Macromol 124:148-154. https://doi.org/10.1016/j.ijbiomac.2018.11.101

70. Cai Z, Dai Q, Guo Y et al (2019) Glycyrrhiza polysaccharide-mediated synthesis of silver nanoparticles and their use for the preparation of nanocomposite curdlan antibacterial film. Int J Biol Macromol 141:422-430. https://doi.org/10.1016/j.jbiomac.2019.09.018

71. Zangheri M, Di Nardo F, Calabria D et al (2021) Smartphone biosensor for point-of-need chemiluminescence detection of ochratoxin a in wine and coffee. Anal Chim Acta 1163:338515. https://doi. org/10.1016/j.aca.2021.338515

72. Choi CK, Shaban SM, Moon BS et al (2021) Smartphone-assisted point-of-care colorimetric biosensor for the detection of urea via pH-mediated AgNPs growth. Anal Chim Acta 1170:338630. https://doi.org/10.1016/J.ACA.2021.338630

73. Liu JM, Wang XX, Jiao $L$ et al (2013) Ultra-sensitive non-aggregation colorimetric sensor for detection of iron based on the signal amplification effect of $\mathrm{Fe}^{3+}$ catalyzing $\mathrm{H}_{2} \mathrm{O}_{2}$ oxidize gold nanorods. Talanta 116:199-204. https://doi.org/10.1016/J.TALANTA.2013.05.024

74. Kumar KS, Ramakrishnappa T (2021) Green synthesized uncapped Ag colloidal nanoparticles for selective colorimetric sensing of divalent $\mathrm{Hg}$ and $\mathrm{H}_{2} \mathrm{O}_{2}$. J Environ Chem Eng 9:105365. https://doi. org/10.1016/J.JECE.2021.105365

75. Yang W, Weng C, Li X et al (2021) A sensitive colorimetric sensor based on one-pot preparation of h- $\mathrm{Fe}_{3} \mathrm{O}_{4} @$ ppy with high peroxidase-like activity for determination of glutathione and $\mathrm{H}_{2} \mathrm{O}_{2}$. Sens Actuators B Chem 338:129844. https://doi.org/10.1016/J.SNB.2021.129844

76. Hou J, Jia P, Yang K et al (2021) Facile preparation of Ru@V2O4 nanowires exhibiting excellent tetra-enzyme mimetic activities for sensitive colorimetric $\mathrm{H}_{2} \mathrm{O}_{2}$ and cysteine sensing. Sens Actuators B Chem 344:130266. https://doi.org/10.1016/J.SNB.2021.130266

77. Liu MM, Li SH, Huang DD et al (2020) MoOx quantum dots with peroxidase-like activity on microfluidic paper-based analytical device for rapid colorimetric detection of $\mathrm{H}_{2} \mathrm{O}_{2}$ released from PC12 cells. Sens Actuators B Chem 305:127512. https://doi.org/10.1016/J.SNB.2019.127512

78. Chen P, Zhong H, Li XR et al (2021) Palygorskite@ $\mathrm{Co}_{3} \mathrm{O}_{4}$ nanocomposites as efficient peroxidase mimics for colorimetric detection of $\mathrm{H}_{2} \mathrm{O}_{2}$ and ascorbic acid. Appl Clay Sci 209:106109. https://doi. org/10.1016/J.CLAY.2021.106109

79. Guo T, Xu T, Xia W et al (2021) Graphene oxide and $\mathrm{CuO}$ double quantum dot composites (GOQD$\mathrm{q}-\mathrm{CuO}$ ) with enhanced haloperoxidase-like activity and its application in colorimetric detection of $\mathrm{H}_{2} \mathrm{O}_{2}$ and glucose. Mater Chem Phys 260:124126. https://doi.org/10.1016/J.MATCHEMPHYS. 2020.124126

80. Jiao X, Song $\mathrm{H}$, Zhao $\mathrm{H}$ et al (2012) Well-redispersed ceria nanoparticles: promising peroxidase mimetics for $\mathrm{H}_{2} \mathrm{O}_{2}$ and glucose detection. Anal Methods. https://doi.org/10.1039/c2ay25511a

81. Chen J, Shu Y, Li H et al (2018) Nickel metal-organic framework 2D nanosheets with enhanced peroxidase nanozyme activity for colorimetric detection of $\mathrm{H}_{2} \mathrm{O}_{2}$. Talanta 189:254-261. https://doi.org/ 10.1016/J.TALANTA.2018.06.075 
82. Liu H, Jiao M, Gu C, Zhang M (2018) Au@CuxOS yolk-shell nanomaterials with porous shells act as a new peroxidase mimic for the colorimetric detection of $\mathrm{H}_{2} \mathrm{O}_{2}$. J Alloys Compd 741:197-204. https://doi.org/10.1016/J.JALLCOM.2017.12.354

83. Ding Y, Yang B, Liu H et al (2018) FePt-Au ternary metallic nanoparticles with the enhanced peroxidase-like activity for ultrafast colorimetric detection of $\mathrm{H}_{2} \mathrm{O}_{2}$. Sens Actuators B Chem 259:775783. https://doi.org/10.1016/J.SNB.2017.12.115

84. Bian B, Liu Q, Yu S (2019) Peroxidase mimetic activity of porphyrin modified $\mathrm{ZnFe}_{2} \mathrm{O}_{4} /$ reduced graphene oxide and its application for colorimetric detection of $\mathrm{H}_{2} \mathrm{O}_{2}$ and glutathione. Colloids Surf B Biointerfaces 181:567-575. https://doi.org/10.1016/J.COLSURFB.2019.06.008

85. Liu G, Liu $\mathrm{H}, \mathrm{Xu} \mathrm{H}$ et al (2020) Enhanced peroxidase-like activity of $\mathrm{Fe}_{3} \mathrm{O}_{4}$-sodium lignosulfonate loaded copper peroxide composites for colorimetric detection of $\mathrm{H}_{2} \mathrm{O}_{2}$ and glutathione. Spectrochim Acta Part A Mol Biomol Spectrosc 239:118544. https://doi.org/10.1016/J.SAA.2020.118544

86. Temur E, Eryiğit M, Kurt Urhan B et al (2021) Cu/Electrochemically reduced graphene oxide layered nanocomposite for non-enzymatic $\mathrm{H}_{2} \mathrm{O}_{2}$ sensor. Mater Today Proc. https://doi.org/10.1016/j. matpr.2021.03.273

87. Banavath R, Srivastava R, Bhargava P (2021) Improved non-enzymatic $\mathrm{H}_{2} \mathrm{O}_{2}$ sensors using highly electroactive cobalt hexacyanoferrate nanostructures prepared through EDTA chelation route. Mater Chem Phys 267:124593. https://doi.org/10.1016/j.matchemphys.2021.124593

Publisher's Note Springer Nature remains neutral with regard to jurisdictional claims in published maps and institutional affiliations.

\section{Authors and Affiliations}

\section{Ezgi $\operatorname{Tan}^{1} \cdot$ İbrahim Mizan Kahyaoğlu ${ }^{2} \cdot$ Selcan Karakuş ${ }^{1}$}

1 Department of Chemistry, Faculty of Engineering, Istanbul University-Cerrahpasa, Avcilar, 34320 Istanbul, Turkey

2 Department of Chemistry, Faculty of Science and Arts, Kurupelit, Ondokuz Mayis University, 55139 Samsun, Turkey 